\title{
Wilson loops stability in the gauge/string correspondence
}

\author{
Raúl E. Arias and Guillermo A. Silva \\ IFLP-CONICET and Departamento de Física \\ Facultad de Ciencias Exactas, Universidad Nacional de La Plata \\ CC 67, 1900, La Plata, Argentina
}

\begin{abstract}
We study the stability of some classical string worldsheet solutions employed for computing the potential energy between two static fundamental quarks in confining and non-confining gravity duals. We discuss the fixing of the diffeomorphism invariance of the string action, its relation with the fluctuation orientation and the interpretation of the quark mass substraction worldsheet needed for computing the potential energy in smooth (confining) gravity background. We consider various dual gravity backgrounds and show by a numerical analysis the existence of instabilities under linear fluctuations for classical string embedding solutions having positive length function derivative $L^{\prime}\left(r_{0}\right)>0$. Finally we make a brief discussion of 't Hooft loops in non-conformal backgrounds.
\end{abstract}




\section{Introduction}

The proof of confinement in non-abelian gauge theories from first principles remains to date unsolved. The strong-coupling aspect of the phenomenon precludes from attacking it with standard QFT perturbative techniques. Nevertheless a criteria for confinement was proposed long ago by Wilson [1]. The criteria states that an area law behavior for the so called Wilson loop indicates confinement of the chromoelectric flux tubes (QCD string). In particular, an area law behavior for a rectangular (infinite strip) spacetime contour corresponding to a static quark-antiquark pair indicates a linear confining potential between quarks.

In the latest years, the gauge/string correspondence [2] has provided new insights into the confinement phenomenon. The crucial observation is that the string aspect of the chromoelectric flux tube manifests in the dual perspective by the appearance of a holographic dimension [2],[3]. A string theory prescription for computing Wilson loops was proposed in [4]: the gauge theory loop is to be though at infinity in the radial holographic coordinate and the Wilson loop for fundamental quarks is defined by an open string whose endpoints lie on the loop at infinity. In the large $N_{c}$ limit ('t Hooft limit) the QCD string self-interactions vanish [6] and the dual gravity prescription for computing the potential energy between quarks in a given gauge theory amounts to finding a minimal surface in the corresponding gravity dual.

The canonical computation for the potential energy between a pair of fundamental static quarks (rectangular loop) involves a U-shaped string extending in the holographic direction where the gauge theory quarks separation $L$ translates into the separation between the fixed open string endpoints located at infinity. As the quarks separation varies, the string worldsheet explores the holographic direction, therefore, the minimum radial position $r_{0}$ reached by the string depend on the endpoints separation distance $L$. This procedure was applied to a number of paradigmatic examples and gave results consistent with gauge theory expectations, in particular a theorem stating sufficient conditions for confining backgrounds was proved in [8] (see [7] for a review). Extensions to higher gauge group representations and 't Hooft loops were analyzed and proposed in [9, [10, [11], they involve higher dimensional $D$-branes with or without worldvolume gauge fields turned on.

In many applications to dual gravity backgrounds the prescription [4 has been applied at the classical zeroth order level to establish confinement, phase transitions or transport properties [7], [12], only recently has the stability of some classical string embeddings been studied [13], [14],[15] (see also [17,, [18], [19],[20]). One of the motivations for the stability analysis, in generalized situations, has been the appearance of multiple classical embedding solutions for given boundary conditions [14, [15, 21] (see also [22]) signaled by the presence of extrema in the length function $L\left(r_{0}\right)$ (see [23] for related recent work). The presence of a maximum separation length was interpreted as dual to the occurrence of screening. The aim of the present work is to show that whenever one has a $L^{\prime}\left(r_{0}\right)>0$ branch of solutions, they are unstable. We will confirm this statement by explicitly showing the existence, in particular gravity backgrounds, of unstable $\left(\omega^{2}<0\right)$ modes for the $L^{\prime}\left(r_{0}\right)>0$ branches. It is worth mentioning that this is a satisfying result since the expected physical behavior for the $L\left(r_{0}\right)$ relation from the gauge/string correspondence is to have $L^{\prime}\left(r_{0}\right)<0$. We will mention briefly an analysis of 't Hooft loops computations in non-conformal gravity duals where instabilities also arise.

Along the way we will discuss various aspects of the classical embeddings: the first one regards the physical interpretation of the configuration employed for obtaining a finite potential energy between quarks in smooth gravity backgrounds, the second regards the diffeomorphism invariance of the string action and its relation to the possible gauge choices for the orientation of the in-plane fluctuations, the third one is the relation between instabilities of the string embedding and the $L\left(r_{0}\right)$ relation between the separation of the string endpoints at infinity $L$ and the maximum depth reached by the string probe in the holographic direction $r_{0}$. It was proved in [15] that the presence of an extremum in the $L\left(r_{0}\right)$ relation leads to the existence of a zero mode for the longitudinal fluctuations, signaling an instability. We will confirm this fact by explicitly computing the lowest fluctuation modes in a number of gravity duals examples.

The paper is organized as follows: in section 2 we review the prescription for computing Wilson loops from gravity backgrounds. In section 3 we describe the backgrounds we will study and compute their length and energy functions. In section 4 we perform a quadratic fluctuations analysis and compute numerically the lowest fluctuation modes. In section 5 we reanalyze the result of section 4 transforming the fluctuations equations of motion into a Schrodinger problem. In section 6 we briefly discuss the 't Hooft loop case and 
in section 7 we summarize our conclusions. We conclude with two appendices with technical details.

\section{Wilson loops and string solutions}

\subsection{Static string U-shaped embeddings}

The starting point for Wilson loop computations, in the large $N_{c}, \lambda$ ('t Hooft limit), from gravity duals with metric $g_{\mu \nu}$ is the Nambu-Goto action 1

$$
S=\frac{\eta}{2 \pi \alpha^{\prime}} \int d \tau d \sigma \sqrt{\eta h} .
$$

Here $h=\operatorname{det} h_{\alpha \beta}, h_{\alpha \beta}=g_{\mu \nu} \partial_{\alpha} X^{\mu} \partial_{\beta} X^{\nu}$ is the induced metric on the string worldsheet, $\partial_{\alpha}=\partial / \partial \xi^{\alpha}$ with $\xi^{\alpha}=\{\tau, \sigma\}$ the string worldsheet coordinates and $X^{\mu}$ run over the target space coordinates. A sign $\eta$ accounts for possible Euclidean $(\eta=+)$ and (timelike) Lorentzian $(\eta=-)$ configurations. The class of metrics we consider take the form

$$
d s^{2}=-g_{t}(r) d t^{2}+g_{x}(r) d x_{i}^{2}+g_{r}(r) d r^{2}+g_{a b}(r, \theta) d \theta^{a} d \theta^{b} .
$$

The $t, x_{i}(i=1,2,3)$ coordinates represent the gauge theory coordinates, $r$ is the bulk holographic coordinate and $\theta_{a}(a, b=1, . ., 5)$ are additional angular coordinates parametrizing a compact 5 d space $\Sigma_{5}$. The potential energy between quarks involves solving the NG action for (timelike) worldsheets corresponding to strings whose endpoints at infinity lie on the loop to be computed, typically the endpoints are kept separated by a constant distance $L$ in one of the $x_{i}$ coordinates which we call $x$ (see [7] for a review). We start analyzing static embeddings of the form $t(\tau), x(\sigma), r(\sigma)$, with all other coordinates fixed to constant. 2 . Placing the anzats into the action leads to the correct equations of motion, one therefore has

$$
\begin{aligned}
S & =-\frac{1}{2 \pi \alpha^{\prime}} \int d \tau d \sigma \sqrt{g_{t}(r) \dot{t}^{2}\left(g_{x}(r) \dot{x}^{2}+g_{r}(r) \dot{r}^{2}\right)} \\
& =-\frac{1}{2 \pi \alpha^{\prime}} \int d t d \sigma \sqrt{g_{t}(r)\left(g_{x}(r) \dot{x}^{2}+g_{r}(r) \dot{r}^{2}\right)} \\
& =-\frac{\mathcal{T}}{2 \pi \alpha^{\prime}} \int d \sigma \sqrt{f^{2}(r) \dot{x}^{2}+g^{2}(r) \dot{r}^{2}}
\end{aligned}
$$

where $g^{2}(r)=g_{t}(r) g_{r}(r)$ and $f^{2}(r)=g_{t}(r) g_{x}(r)$. The reparametrization invariance of (1) factorizes the temporal extension of the loop $\mathcal{T}$ and reduces the Wilson loop computation to finding a geodesic in the effective 2-dimensional geometry

$$
d s_{\text {eff }}^{2}=f^{2}(r) d x^{2}+g^{2}(r) d r^{2} .
$$

The conserved charge associated to $x$-translations in (3) is

$$
\frac{f^{2}(r) \dot{x}(\sigma)}{\sqrt{f^{2}(r) \dot{x}(\sigma)^{2}+g^{2}(r) r^{\prime}(\sigma)^{2}}}=A
$$

from which one obtains

$$
\dot{x}(\sigma)= \pm A \frac{g(r)}{f(r)} \frac{1}{\sqrt{f^{2}(r)-A^{2}}} \dot{r}(\sigma) .
$$

Reparametrization invariance guarantees that (6) solves the $r$-equation of motion. Calling $r_{0}$ the point given by $f\left(r_{0}\right)=A$, (6) can rewritten as [8]

$$
\frac{d x}{d r}= \pm \frac{g(r)}{f(r)} \frac{f\left(r_{0}\right)}{\sqrt{f^{2}(r)-f^{2}\left(r_{0}\right)}} .
$$

\footnotetext{
${ }^{1}$ Generically one should take into account contributions from $B_{2}$ background fields but for the ansatz we will consider they do not contribute to (1).

${ }^{2}$ Generalizations considering moving quarks on the boundary, relevant for QGP applications, are straightforward and rephrased in terms of non-diagonal terms in the metric [14, 21.
} 
The boundary conditions at infinity for the string endpoints separation are $\left.\Delta x\right|_{r=\infty}=L$. From (7) one notices that the string reaches the boundary in an orthogonal way. Two natural gauge choices that appear in the literature are: $x(\sigma)=\sigma$ (x-gauge) or $r(\sigma)=\sigma$ (r-gauge). The first choice ( $x$-gauge) has the benefit of providing a complete parametrization of the embedding $r(x)$ when imposing $x \in[-L / 2, L / 2]$ and $r( \pm L / 2)=\infty$ (the tip of the string is conventionally chosen to be at $(r, x)=\left(r_{0}, 0\right)$ ). Making $x \rightarrow t$, equation (7) can be understood as a zero energy motion in a potential $U(r)$ given by

$$
U(r)=\frac{f^{2}(r)\left(f^{2}(r)-f^{2}\left(r_{0}\right)\right)}{g^{2}(r) f^{2}\left(r_{0}\right)}
$$

the point $r_{0}$ is therefore easily seen to be the minimum value in the holographic coordinate reached by the string. The second choice ( $r$-gauge) gives a double valued $x(r)$ relation when imposing $r \in\left[r_{0}, \infty\right)$ and $x(\infty)= \pm L / 2$. Nevertheless in several examples leads to closed analytical expressions for $x(r)$ and moreover, when computing fluctuations around the static solution, drastically simplify the equations of motion since no fluctuations in the metric components $g_{\mu \nu}$ should be taken into account (see [8], [15]). One should keep in mind that the tip of the string is a special point in the $r$-gauge since we must patch there the two branches corresponding to the \pm signs in (7) (see the following section).

Integrating (6) we arrive to the important $L\left(r_{0}\right)$ length function,

$$
L\left(r_{0}\right)=2 \int_{r_{0}}^{\infty} \frac{g(r)}{f(r)} \frac{f\left(r_{0}\right)}{\sqrt{f^{2}(r)-f^{2}\left(r_{0}\right)}} d r .
$$

Assuming that $f(r), f^{\prime}(r)>0$ the lower limit $r_{0}$ in (9) is generically integrable 3 . Note that a finite lhs in (9) demands that $g / f^{2}$ should decay at infinity faster than $1 / r$. In the following sections we will be interested in the relation (9). The derivative $L^{\prime}\left(r_{0}\right)$ can be computed as follows [15] (see also the recent work [33]),

$$
\begin{aligned}
\frac{L^{\prime}\left(r_{0}\right)}{2} & =-\left.\frac{g(r)}{\sqrt{f^{2}(r)-f^{2}\left(r_{0}\right)}}\right|_{r \rightarrow r_{0}}+f^{\prime}\left(r_{0}\right) \int_{r_{0}}^{\infty} \frac{f(r) g(r)}{\left(f^{2}(r)-f^{2}\left(r_{0}\right)\right)^{\frac{3}{2}}} d r \\
& =-\left.\frac{g(r)}{\sqrt{f^{2}(r)-f^{2}\left(r_{0}\right)}}\right|_{r \rightarrow r_{0}}+f^{\prime}\left(r_{0}\right) \int_{r_{0}}^{\infty} d r \frac{g(r)}{f^{\prime}(r)} \frac{d}{d r}\left(-\frac{1}{\sqrt{f^{2}(r)-f^{2}\left(r_{0}\right)}}\right) \\
& =-\left.\frac{f^{\prime}\left(r_{0}\right) g(r)}{f^{\prime}(r) \sqrt{f^{2}(r)-f^{2}\left(r_{0}\right)}}\right|_{r \rightarrow \infty}+\int_{r_{0}}^{\infty} d r \frac{f^{\prime}\left(r_{0}\right)}{\sqrt{f^{2}(r)-f^{2}\left(r_{0}\right)}} \frac{d}{d r}\left(\frac{g(r)}{f^{\prime}(r)}\right),
\end{aligned}
$$

where we have integrated by parts when passing from the second to the third line. Since the first term in the rhs of the third line vanishes in all the backgrounds we consider, we obtain

$$
L^{\prime}\left(r_{0}\right)=2 \int_{r_{0}}^{\infty} d r \frac{f^{\prime}\left(r_{0}\right)}{\sqrt{f^{2}(r)-f^{2}\left(r_{0}\right)}} \frac{d}{d r}\left(\frac{g(r)}{f^{\prime}(r)}\right) .
$$

The energy of the $q \bar{q}$ configuration was proposed in [4] to be given by the length of the string solution (6) in the effective $2 \mathrm{~d}$ metric (44),

$$
E=\frac{1}{2 \pi \alpha^{\prime}} \int d \sigma \sqrt{f^{2}(r) \dot{x}(\sigma)^{2}+g^{2}(r) \dot{r}(\sigma)^{2}}
$$

The expressions for the energy in the $x$ - and $r$-gauges, using (6) are

$$
\begin{aligned}
E\left(r_{0}\right) & =\frac{1}{2 \pi \alpha^{\prime}} \int_{-L / 2}^{L / 2} d x \frac{f^{2}(r(x))}{f\left(r_{0}\right)} \\
& =\frac{1}{\pi \alpha^{\prime}} \int_{r_{0}}^{\infty} d r \frac{g(r) f(r)}{\sqrt{f^{2}(r)-f^{2}\left(r_{0}\right)}}
\end{aligned}
$$

\footnotetext{
${ }^{3}$ A zero $f^{\prime}\left(r_{0}\right)=0$ leads to a $r_{0}$-dependent logarithmic divergence in (9) (see [33] for a generic discussion on divergences in the length function).
} 
The energy computed by expression (11) diverges due to the infinite extension of the stringt The interpretation for this divergence is that (11) contains, in addition to the potential energy between quarks, the self energy (mass) of the external quarks [4]. In order to obtain a meaningful quantity and get the potential between quarks we should compare (11) with respect to a reference state taking care to substract a $r_{0}$ independent quantity. It is customary to take the length of a straight string stretching from infinity all the way down to the interior of the bulk spacetime along the $r$ coordinate, with all other coordinates set to constants, as the "bare" quark mass. Calling $r=r_{\min }$ the minimum allowed value for the radial coordinate in the geometry (2), either because of presence of a horizon (e.g. AdS in Poincare coordinates or thermal BH backgrounds) or because the spacetime ends in a regular fashion (e.g. Witten $A d S$ soliton, Maldacena Núñez and Klebanov-Strassler backgrounds), the quark mass takes the form 5

$$
m_{q}=\frac{1}{2 \pi \alpha^{\prime}} \int_{r_{\text {min }}}^{\infty} g(r) d r .
$$

The potential energy between quarks obtained from (11) after substracting the quarks self energy (14) is

$$
\begin{aligned}
E_{q \bar{q}}\left(r_{0}\right) & =E\left(r_{0}\right)-2 m_{q} \\
& =\frac{1}{\pi \alpha^{\prime}}\left[\int_{r_{0}}^{\infty} \frac{g(r) f(r)}{\sqrt{f^{2}(r)-f^{2}\left(r_{0}\right)}} d r-\int_{r_{\text {min }}}^{\infty} g(r) d r\right] .
\end{aligned}
$$

Eliminating $r_{0}$ from (9) and (15) we obtain the gauge/string proposal for the potential energy between quarks in the planar large 't Hooft limit $V_{\text {string }}(L)$. In the following sections we will plot this relation in several examples and analyze its functional form. For completeness we compute the derivative of (15), one has

$$
E_{q \bar{q}}^{\prime}\left(r_{0}\right)=\frac{1}{\pi \alpha^{\prime}}\left[-\left.\frac{g(r) f(r)}{\sqrt{f^{2}(r)-f^{2}\left(r_{0}\right)}}\right|_{r=r_{0}}+\int_{r_{0}}^{\infty} d r \frac{f(r) g(r) f\left(r_{0}\right) f^{\prime}\left(r_{0}\right)}{\left(f^{2}(r)-f^{2}\left(r_{0}\right)\right)^{\frac{3}{2}}}\right] .
$$

Using the first line of (10) one obtains [15]

$$
E_{q \bar{q}}^{\prime}\left(r_{0}\right)=\frac{1}{2 \pi \alpha^{\prime}} f\left(r_{0}\right) L^{\prime}\left(r_{0}\right) \Rightarrow \frac{d E_{q \bar{q}}}{d L}=\frac{1}{2 \pi \alpha^{\prime}} f\left(r_{0}\right),
$$

where $r_{0}$ in the last expression should be understood as the function $r_{0}(L)$ obtained by inverting (9).

We end this section quoting some conditions that must be satisfied by any potential pretending to describe the interaction between physical quarks. The so called 'concavity' conditions proved in 24] are

$$
\frac{d V}{d L}>0, \quad \frac{d^{2} V}{d L^{2}} \leq 0 .
$$

This conditions hold independently of the gauge group and the details of the matter sector. The physical interpretation of (17) is that the force between the quark-antiquark pair is: (i) always attractive and (ii) a non increasing function of their separation distance. From (9), (15), (16) we find that the string proposal $V_{\text {string }}(L)$ gives [22]

$$
\frac{d V_{\text {string }}}{d L}=\frac{d E_{q \bar{q}}}{d r_{0}} \frac{d r_{0}}{d L}=\frac{1}{2 \pi \alpha^{\prime}} f\left(r_{0}\right), \quad \frac{d^{2} V_{\text {string }}}{d L^{2}}=\frac{1}{2 \pi \alpha^{\prime}}\left(\frac{d L}{d r_{0}}\right)^{-1} f^{\prime}\left(r_{0}\right) .
$$

The first condition is always met in dual gravity backgrounds since by definition $f(r)>0$. Although in all our examples $f^{\prime}(r)>0$, the second condition might fail whenever $L^{\prime}\left(r_{0}\right)$ is positive. We will present cases where this non-physical behavior appears and show that precisely in those circumstances the string embedding solution (7)-(9) is unstable under small perturbations. This last statement was the motivation of the present work.

\footnotetext{
${ }^{4}$ The divergence in (13) is generically $r_{0}$-independent, an additional $r_{0}$-dependent divergence might appear in (9) and (11) whenever the string stretches to regions where $f^{\prime}\left(r_{0}\right)=0$.

${ }^{5}$ See the discussion at the end of sections 3.1 .2 and 3.3 regarding the interpretation of the reference state in smooth gravity backgrounds.
} 


\subsection{Stability analysis of classical string embeddings}

We will study in this section the stability of the classical solution $\left(r_{\mathrm{cl}}(\sigma), x_{\mathrm{cl}}(\sigma)\right)$ given by (7)-(9) under small (linear) perturbations. A general fluctuation around the embedding solution can be written as

$$
X^{\mu}=\left(\tau, x_{\mathrm{cl}}(\sigma)+\delta x_{1}(\tau, \sigma), \delta x_{2}(\tau, \sigma), \delta x_{3}(\tau, \sigma), r_{\mathrm{cl}}(\sigma)+\delta r(\tau, \sigma), \theta^{a}+\delta \theta^{a}(\tau, \sigma)\right) .
$$

We can use the diffeomorphism invariance of the action to fix $t=\tau$ and forget about the $t$-equation of motion. For the class of metrics considered in (2), the $\delta x_{2}$ and $\delta x_{3}$ fluctuations decouple and as expected satisfy the same equation of motion, the $\delta \theta^{a}$ fluctuations mix among themselves for generic compact manifolds and lead to five eom (we will not analyze the angular fluctuations in the present work and we consistently set them to zero), finally the $\delta x_{1}$ and $\delta r$ fluctuations result mixed in two coupled equations.

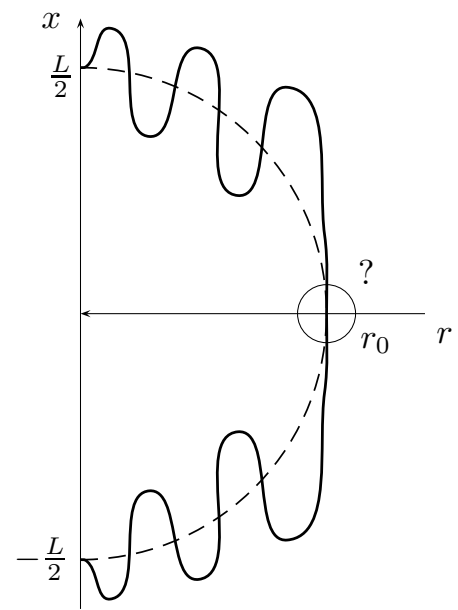

Figure 1: $r$-gauge fixing: The dashed line represents the classical embedding over which we perturb. At the tip $r_{0}$, the fluctuation is oriented along the string, and therefore not physical.

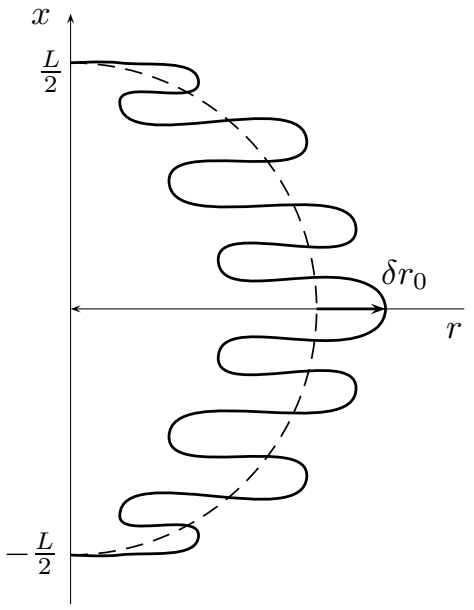

Figure 2: $x$-gauge fixing: The dashed line represents the classical embedding we are perturbing. This is a physical gauge choice all over the embedding solution, the plotted (even) fluctuation changes the position of the tip.

It is easily shown that the $r$ - and $x_{1}$-equations of motion are proportional. The remaining diffeomorphism should therefore be used to fix the orientation of the $\left(\delta r, \delta x_{1}\right)$ vector (in-plane fluctuation) at each point of the solution $\left(r_{\mathrm{cl}}(\sigma), x_{\mathrm{cl}}(\sigma)\right)$. After imposing a gauge constraint one equation describes the fluctuations in the $\left(r, x_{1}\right)$-plane and we end up with a well posed system of differential equations. The physical gauge choice ( $n$-gauge) would be to orient the fluctuation along the normal direction to the classical embedding $\left(r_{\mathrm{cl}}(\sigma), x_{\mathrm{cl}}(\sigma)\right)$ and generically this means to account for fluctuations on both $x_{1}$ and $r$ coordinates. Two other natural possibilities considered in the literature correspond to fixing $\delta x_{1}(\tau, \sigma)=0$ (x-gauge) or $\delta r(\tau, \sigma)=0$ ( $r$-gauge). The $n$ - and $x$-gauge fixings, as mentioned in the previous section, parametrize the fluctuations along the whole classical embedding but the equations of motion are lengthier because the $\delta r$ fluctuations result in additional contributions to the eom arising from changes in the metric [18, [15]. Note that at first sight the $n$ - and $x$-gauge fixings appear to allow for the oscillation of the tip, while the $r$-gauge fixing should not (more on this in the following). We will choose to work in the $r$-gauge, this means fixing $\delta r(\tau, \sigma)=0$ and work with simpler equations defined on half of the embedding. It will then be mandatory to analyze the boundary conditions to be imposed at the tip $\left(r_{0}, 0\right)$ of the embedding in order to get a meaningful solution. Moreover, since we are considering fluctuations along the $x_{1}$ coordinate, precisely at the tip the $\delta x_{1}$ fluctuation is oriented along the string worldvolume and not transverse to it: the $\delta x_{1}$ fluctuation in the $r$-gauge is therefore not physical at the tip of the embedding. The required additional analysis developed in [14, [15] will be discussed below. At last, another advantage of the $r$-gauge is that it gives closed expressions 
for the linearized fluctuations equations of motion (see (22)-(23)), the isometry along $x_{1}$ implies that upon computing fluctuations over the classical solution (7)-(9), we do not need the explicit analytic solution $x_{\mathrm{cl}}(r)$, what contribute to the equations of motion is its derivative (7) (as an example compare (113) with (114)). We would like to quote the work [14] where $\delta r$ fluctuations in the $\delta x_{1}=0$ gauge fixing were considered over (half) the classical embedding parametrized in the $r$-gauge (see below eqn. (27),(28)).

In the following we will be concerned with the equations of motion for the $\delta x_{i}$ fluctuations, they can be obtained by placing the ansatz

$$
t=\tau, \quad x_{1}=x_{\mathrm{cl}}(r)+\delta x_{1}(t, r), \quad x_{2}=\delta x_{2}(t, r), \quad x_{3}=\delta x_{3}(t, r), \quad r=\sigma,
$$

into the action (1). Expanding to second order in the fluctuations one obtains

$$
\begin{aligned}
2 \pi \alpha^{\prime} \mathcal{L}^{(2)}= & \frac{1}{g(r) f(r) \sqrt{f^{2}(r)-f^{2}\left(r_{0}\right)}}\left[h^{2}(r)\left(f^{2}(r)-f^{2}\left(r_{0}\right)\right)\left(\delta \dot{x}_{1}\right)^{2}-\left(f^{2}(r)-f^{2}\left(r_{0}\right)\right)^{2}\left(\delta \dot{x}_{1}\right)^{2}\right. \\
& \left.+f^{2}(r) h^{2}(r)\left(\left(\delta \dot{x}_{2}\right)^{2}+\left(\delta \dot{x}_{3}\right)^{2}\right)-f^{2}(r)\left(f^{2}(r)-f^{2}\left(r_{0}\right)\right)\left(\left(\delta \dot{x}_{2}\right)^{2}+\left(\delta \dot{x}_{3}\right)^{2}\right)\right]
\end{aligned}
$$

where $h^{2}(r)=g_{x}(r) g_{r}(r)$. The Euler-Lagrange equation for the $\delta x_{1}$ fluctuation is

$$
\left[\frac{d}{d r}\left(\frac{\left(f^{2}(r)-f^{2}\left(r_{0}\right)\right)^{\frac{3}{2}}}{g(r) f(r)} \frac{d}{d r}\right)+\omega^{2} \frac{h^{2}(r) \sqrt{f^{2}(r)-f^{2}\left(r_{0}\right)}}{g(r) f(r)}\right] \delta x_{1}(r)=0,
$$

where we factorized the time dependence of the fluctuations as $\delta x(t, r)=\delta x(r) e^{-i \omega t}$. The equations for the fluctuations transverse to the $\left(r, x_{1}\right)$-plane obtained from (21) are

$$
\left[\frac{d}{d r}\left(\frac{f(r) \sqrt{f^{2}(r)-f^{2}\left(r_{0}\right)}}{g(r)} \frac{d}{d r}\right)+\omega^{2} \frac{h^{2}(r) f(r)}{g(r) \sqrt{f^{2}(r)-f^{2}\left(r_{0}\right)}}\right] \delta x_{m}(r)=0, \quad m=2,3 .
$$

Equations (22)-(23) are differential equations of the Sturm-Liouville type defined for the half line $r_{\min } \leq$ $r_{0} \leq r<\infty$ and we are interested in analyzing the existence of instabilities, in particular determining the range of values of $r_{0}$ for which $\omega^{2}<0$.

The boundary conditions to be imposed on the problem are Dirichlet, this means fluctuations keeping the string endpoints fixed at the boundary $\left.\delta x_{i}(\tau, \sigma)\right|_{r=\infty}=0$, but, the nature of the $r$-gauge parametrizing only half of the $\left(r_{\mathrm{cl}}(\sigma), x_{\mathrm{cl}}(\sigma)\right)$ embedding requires an additional analysis of boundary conditions at the tip $r=r_{0}$ (singular point of (22)-(23) $)$. We start by analyzing (23), the expansion around the tip gives 6

$\frac{d}{d r}\left(\sqrt{r-r_{0}} \frac{d \delta x_{m}(r)}{d r}\right)+\frac{\omega^{2} h^{2}\left(r_{0}\right)}{2 f\left(r_{0}\right) f^{\prime}\left(r_{0}\right)} \frac{1}{\sqrt{r-r_{0}}} \delta x_{m}(r) \approx 0 \quad \Longrightarrow \quad \delta x_{m}(r) \approx C_{0}+C_{1} \sqrt{r-r_{0}}+O\left(r-r_{0}\right)$.

Here $C_{0,1}$ are arbitrary constants corresponding to the two independent solutions of the differential equation (23), which once chosen determine the whole series expansion for $\delta x_{m}(r)$. Physically they correspond respectively to even and odd fluctuations around the tip once we patch them with the fluctuations around the other half of the embedding, which obviously satisfy the same eom. A discrete set of eigenvalues $\omega^{2}$ is expected if non-normalizable solutions exist in the large $r$-limit, we also expect the even solution to have the lowest $\omega^{2}$ eigenvalue (see appendix B).

We now turn to the analysis of the in-plane $\delta x_{1}$ fluctuation. Expanding (22) around $r=r_{0}$ one finds

$\frac{d}{d r}\left(\left(r-r_{0}\right)^{\frac{3}{2}} \frac{d \delta x_{1}(r)}{d r}\right)+\frac{\omega^{2} h^{2}\left(r_{0}\right)}{2 f\left(r_{0}\right) f^{\prime}\left(r_{0}\right)} \sqrt{r-r_{0}} \delta x_{1}(r) \approx 0 \quad \Longrightarrow \quad \delta x_{1}(r) \approx C_{0}^{\prime}+C_{1}^{\prime} \frac{1}{\sqrt{r-r_{0}}}+O\left(\sqrt{r-r_{0}}\right)$.

A singular behavior appears for $\delta x_{1}$ at the tip and one might be tempted to cancel it by imposing $C_{1}^{\prime}=0$. Nevertheless, as mentioned above one should take notice that the $r$-gauge fixing implies that, at the tip, $\delta x_{1}$ is directed along the string worldsheet and not transverse to it, therefore in the $r$-gauge the displacement

${ }^{6}$ We consider $f(r)$ to be an increasing function of $r$ having no zeroes except perhaps at the bottom of the bulk $r=r_{m i n}$. 
$\delta x_{1}$ at the tip is not physical. In order to give a physical interpretation to the constants $C_{0,1}^{\prime}$ in (25) we now make a change from the $r$ - to the $x$-gauge (cf. [12, 15]). The change of gauge on the ansatz (20) can be implemented by a change of variables on the solution (22) from $r$ to a new variable which we call $u$. It can be implemented perturbatively, to first order in the fluctuation the relation is [15],

$$
u=r+\Delta(t, r) \quad \text { with } \quad \Delta(t, r)=\frac{\delta x_{1}(t, r)}{x_{\mathrm{cl}}^{\prime}(r)} .
$$

This transformation performs the desired change of gauge since

$$
\begin{aligned}
x_{1} & =x_{\mathrm{cl}}(r)+\delta x_{1}(t, r)=x_{\mathrm{cl}}(u-\Delta(t, r))+\delta x_{1}(t, r) \\
& \approx x_{\mathrm{cl}}(u)-x_{\mathrm{cl}}^{\prime}(r) \frac{\delta x_{1}(t, r)}{x_{\mathrm{cl}}^{\prime}(r)}+\delta x_{1}(t, r)=x_{\mathrm{cl}}(u) \\
r & \approx u-\frac{\delta x_{1}(t, u)}{x_{\mathrm{cl}}^{\prime}(u)},
\end{aligned}
$$

here $r_{0} \leq u<\infty$. This is precisely the gauge fixing employed in [14] mentioned above. The second term on the rhs of (28) is interpreted as the $r$-direction fluctuation induced by the ( $r$-gauge) $x_{1}$-fluctuation. It is now easy to see that it is finite. The asymptotic behavior (25) and the expansion around the tip of (7), $x_{\mathrm{cl}}^{\prime}(r) \sim\left(r-r_{0}\right)^{-1 / 2}$, plugged in (28) gives

$$
r \approx r_{0}-\alpha\left(C_{0}^{\prime} \sqrt{u-r_{0}}+C_{1}^{\prime}\right)+O\left(u-r_{0}\right),
$$

with $\alpha$ a finite constant. The result (29) shows that the physical $\delta r$ fluctuation originated from the nonphysical divergent $\delta x_{1}$ fluctuation at the tip is manifestly finite. Therefore we interpret the $\left(C_{0}^{\prime}\right) C_{1}^{\prime}$ in (25) as the fluctuation that (do not) oscillates the tip position.

In the following sections we will show numerical solutions of (22) for various gravity backgrounds determining the lowest eigenvalues leading to normalizable solutions. We will solve (22) by a shooting method integrating numerically from the tip $r_{0}$ up to a large value $r_{\infty}$. The allowed values for $\omega^{2}$ will be obtained imposing the numerical solution to be zero at $r=r_{\infty}$. The boundary conditions at the tip corresponding to even solutions are $C_{0}^{\prime}=0$ and $C_{1}^{\prime}$ arbitrary, for numerical purposes we set $C_{1}^{\prime}=1$, its value sets the normalization of the fluctuation. An even solution around the tip satisfies

$$
\left.\frac{d \delta r(t, r)}{d x_{1}}\right|_{r=r_{0}}=0 \quad \text { where } \quad \delta r(t, r)=-\frac{\delta x_{1}(t, r)}{x_{\mathrm{cl}}^{\prime}(r)} .
$$

Using (27) we can write (30) in terms of $\delta x_{1}(r)$. The boundary conditions for even solutions of (22) are implemented numerically as

$$
\begin{aligned}
\delta x_{1}(r)+2\left(r-r_{0}\right) \frac{d \delta x_{1}(r)}{d r} & =0, & r & \rightarrow r_{0} \\
\sqrt{r-r_{0}} \delta x_{1}(r) & =1, & r & \rightarrow r_{0} .
\end{aligned}
$$

Odd solutions $C_{0}^{\prime}=1$ and $C_{1}^{\prime}=0$ are implemented as

$$
\begin{aligned}
\delta x_{1}(r)+2\left(r-r_{0}\right) \frac{d \delta x_{1}(r)}{d r} & =1, \quad r \rightarrow r_{0} \\
\sqrt{r-r_{0}} \delta x_{1}(r)=0, & r \rightarrow r_{0} .
\end{aligned}
$$

Summarizing, in general backgrounds, the functional relation of the classical solution (7) between the $x_{1}$ and $r$ coordinates at the tip takes the form $x_{\mathrm{cl}}^{2}(r) \approx r-r_{0}$, and the asymptotic behavior of the $x_{1}$-fluctuations over it, in the $r$-gauge, is of the form (25). Although a divergent piece appears in (25), an appropriate change of gauge shows that the divergent and non-divergent pieces correspond respectively to (physical) even and odd fluctuations around the tip. 


\section{Gravity Backgrounds}

In this section we compute the string embeddings (6) dual to rectangular Wilson loops for a a number of paradigmatic gravity backgrounds. We review the $A d S_{5} \times S^{5}[4]$ and $A d S_{5}$-Schwarzschild $\times S^{5}[16]$ cases. Next we perform the numerical analysis of the equations (9) and (15) for the Maldacena-Nuñez [25], KlebanovStrassler [26] and the generalized Maldacena-Nuñez [28] backgrounds. In all cases the geometry is supported by some non-trivial $p$-form fluxes, but they will not be relevant for our computations.

\section{1 $A d S_{5} \times S^{5}$}

This background is dual to $\mathcal{N}=4 \mathrm{SYM}$ with $G=S U(N)$ gauge group in the Coulomb phase. The $A d S$ curvature $R$ relates to the gauge theory 't Hooft coupling $\lambda$ as $R^{4}=\alpha^{\prime 2} \lambda$ and the flux of the 5form supporting the geometry $N=\int_{S^{5}} F_{5}$ relates to the rank of the gauge group as $N=\operatorname{Rank}(G)[2]$. The conformal invariance of the gauge theory implies a Coulomb behavior for the potential $V(L) \sim 1 / L$ between quarks. The novelty of the gravity computation is to compute the gauge coupling dependence of the proportionality coefficient.

\subsubsection{Poincare coordinates [4]}

This coordinate system is supposed to describe the gauge theory formulated on $\mathbb{R}^{3,1}$. The metric is written as

$$
d s^{2}=\frac{r^{2}}{R^{2}}\left(-d t^{2}+d x_{i} d x_{i}\right)+R^{2} \frac{d r^{2}}{r^{2}}+R^{2} d \Omega_{5}^{2} .
$$

One finds $f^{2}(r)=r^{4} / R^{4}, g^{2}(r)=1$. The radial coordinate range is $0<r<\infty$, at $r=0$ one finds a Killing horizon. Equation (7) can be analytically solved in the $r$-gauge, one obtains

$$
x_{\mathrm{cl}}(r)= \pm\left\{c t e-\frac{R^{2}}{4 r_{0}} \mathrm{~B}\left(\left(\frac{r_{0}}{r}\right)^{4} ; \frac{3}{4}, \frac{1}{2}\right)\right\}, \quad r_{0} \leq r<\infty
$$

where $\mathrm{B}(z ; a, b)$ is the incomplete beta function $\mathrm{B}(z ; a, b)=\int_{0}^{z} t^{a-1}(1-t)^{b-1} d t$. The boundary conditions fix the constant in (34) and relate the parameters $r_{0}$ and $L$, setting $x_{\mathrm{cl}}\left(r_{0}\right)=0$ and $x_{\mathrm{cl}}(\infty)= \pm L / 2$ one obtains [4],

$$
L\left(r_{0}\right)=\frac{R^{2}}{2 r_{0}} \mathrm{~B}\left(\frac{3}{4}, \frac{1}{2}\right)=\frac{R^{2}}{r_{0}} \frac{(2 \pi)^{\frac{3}{2}}}{\Gamma\left[\frac{1}{4}\right]^{2}} .
$$

The energy (15) takes the form [4]

$$
E_{q \bar{q}}\left(r_{0}\right)=\frac{r_{0}}{\pi \alpha^{\prime}}(K(-1)-E(-1))=-\frac{r_{0}}{2 \pi \alpha^{\prime}} \frac{(2 \pi)^{\frac{3}{2}}}{\Gamma\left[\frac{1}{4}\right]^{2}},
$$

here $K(\mathrm{~m}), E(\mathrm{~m})$ are the complete elliptic integrals of first and second kind. Eliminating $r_{0}$ from expressions (35)-(36) the AdS/CFT proposal for the interaction potential between fundamental quarks in the large 't Hooft coupling for the $\mathcal{N}=4 \mathrm{SYM}$ theory is [4]

$$
V_{\text {string }}(L)=-\frac{(2 \pi)^{2}}{\Gamma\left[\frac{1}{4}\right]^{4}} \frac{R^{2} / \alpha^{\prime}}{L} \sim-\frac{\sqrt{\lambda}}{L} .
$$

An attractive Coulomb potential is obtained as expected from conformal invariance. The interesting result is the $\sqrt{\lambda}=\left(g_{Y M}^{2} N\right)^{\frac{1}{2}}$ dependence when compared with the perturbative $\lambda=g_{Y M}^{2} N$ result. This suggests that some renormalization of the charges takes place at strong coupling [4]. Note that in order to obtain the negative quantity (37) starting from a positive definite one (see eqn (11)), it was crucial to substract the quark masses (14). 


\subsubsection{Global coordinates}

We discuss this example because it clarifies conceptual issues regarding the interpretation of the substraction procedure (15) in smooth and complete gravity backgrounds (see sections 3.3, 3.4, 3.5).

Computations performed in $A d S$ global coordinates are supposed to represent the $\mathcal{N}=4$ SYM gauge theory defined on $S^{3} \times \mathbb{R}$. The $A d S$ metric is now written as

$$
d s^{2}=R^{2}\left[-\cosh ^{2} \rho d t^{2}+d \rho^{2}+\sinh ^{2} \rho d \Omega_{3}^{2}\right] .
$$

All coordinates are dimensionless in this case with the $A d S$ radius $R$ setting the scale. We write the $S^{3}$ metric as $d \Omega_{3}^{2}=d \theta_{1}^{2}+\sin ^{2} \theta_{1}\left(d \theta_{2}^{2}+\sin ^{2} \theta_{2} d \varphi^{2}\right)$. Being $\varphi$ a cyclic coordinate, the appropriate string ansatz is $t=\tau, \rho=\rho(\sigma), \varphi=\sigma$, one obtains $f^{2}(\rho)=\frac{1}{4} \sinh ^{2} 2 \rho, g^{2}(\rho)=\cosh ^{2} \rho$. The remaining angular variables must be set to $\theta_{i}=\frac{\pi}{2}$ (equator of $S^{3}$ ) in order to satisfy their eom. The conserved charge in the $\varphi$ coordinate leads to an effective one dimensional zero energy motion

$$
\dot{\rho}^{2}+U(\rho)=0,
$$

where $\rho=d \rho / d \varphi$ and the potential $U(\rho)=\sinh ^{2} \rho\left(1-\frac{\sinh ^{2} 2 \rho}{\sinh ^{2} 2 \rho_{0}}\right) \cdot \rho_{0}$ is the minimum radial position reached by the string when separating the string endpoints at infinity by $\Delta \varphi=\Phi$. The $\Phi\left(\rho_{0}\right)$ relation (9) is computed straightforwardly

$$
\Phi\left(\rho_{0}\right)=2 \int_{r_{0}}^{\infty} \frac{\sinh 2 \rho_{0}}{\sinh \rho \sqrt{\sinh ^{2} 2 \rho-\sinh ^{2} 2 \rho_{0}}} d \rho .
$$

Since the gauge theory is defined on a $S^{3}$ there exists a maximum separation for the quarks and it corresponds to placing them at antipodes on the equator of the $S^{3}$. This results in the string reaching the origin $\Phi(0)=\pi$ and leading to a smooth straight worldsheet (stretched along the radial $r$ coordinate) parametrized by two halves at $\varphi=\varphi_{0}$ and $\varphi=\pi+\varphi_{0}$. The (divergent) energy (13) of the configuration (39) is

$$
E\left(\rho_{0}\right)=\frac{R}{2 \pi \alpha^{\prime}} \int_{\rho_{0}}^{\infty} \frac{\sinh ^{2} 2 \rho}{\sinh \rho \sqrt{\sinh ^{2} 2 \rho-\sinh ^{2} 2 \rho_{0}}} d \rho .
$$

Substracting the quark masses as in (15) leads to,

$$
E_{q \bar{q}}\left(\rho_{0}\right)=\frac{R}{2 \pi \alpha^{\prime}}\left[\int_{\rho_{0}}^{\infty}\left(\frac{2 \cosh \rho}{\sqrt{1-\frac{\sinh ^{2} 2 \rho_{0}}{\sinh ^{2} 2 \rho}}}-2 \cosh \rho\right) d \rho-2 \sinh \rho_{0}\right],
$$

which is finite and negative definite (see fig.3). The finite result (42) should be understood as resulting from the comparison of (41) wrt the aforementioned smooth reference state consisting in a straight string with its endpoints at infinity at antipodes on the $S^{3}$ equator. We interpret this last configuration as the one corresponding to "infinitely" separated quarks on $S^{3}$. Note that the reference state and the configuration we are analyzing satisfy different boundary conditions.

\section{$3.2 \quad A d S_{5}$-Schwarzschild $\times S^{5}$}

Finite temperature gauge theories are described by considering black hole $(\mathrm{BH})$ solutions in the gravity duals [5. The near horizon geometry of $N$ non extremal (black) D3-branes is therefore conjectured to describe $\mathcal{N}=4 \mathrm{SYM}$ at finite temperature. As explained in [5], the appropriate $\mathrm{BH}$ background for describing the gauge theory on $\mathbb{R}^{3,1}$ involves a delicate infinite mass limit of the $A d S_{5}$-Schwarzschild BH resulting in 7 ,

$$
d s^{2}=\frac{r^{2}}{R^{2}}\left[-\left(1-\frac{\mu^{4}}{r^{4}}\right) d t^{2}+d x_{i} d x_{i}\right]+\frac{R^{2}}{r^{2}} \frac{1}{1-\frac{\mu^{4}}{r^{4}}} d r^{2}+R^{2} d \Omega_{5}^{2} .
$$

\footnotetext{
${ }^{7}$ The limit results in the metric depending on only one scale, contrary to the finite mass case where one has two parameters: the temperature (BH mass) and the $A d S$ radius. This last geometry was shown to exhibit a phase transition (Hawking-Page), which was interpreted as dual to the confiniement/deconfinement phase transition in $\mathcal{N}=4$ on $S^{3}[5$.
} 


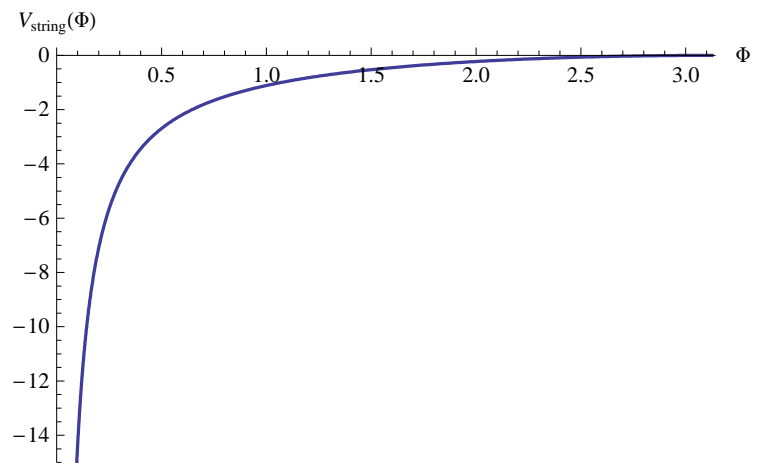

Figure 3: $V_{\text {string }}(\Phi)$ obtained from (40) and (421). For small angular separations between the string endpoints $\Phi \ll 1$ one finds the expected Coulomb behavior $V \sim \sqrt{\lambda} /(R \Phi)$. For larger separations the solution deviates due to the compactness of the $S^{3}$.

The $\mathrm{BH}$ horizon is located at $r=\mu$ and its temperature is $T=\frac{\mu}{R^{2}} \pi$. It is convenient to work with dimensionless coordinates, scaling $r=\mu \rho, t=R^{2} / \mu \bar{t}$ and $x=R^{2} / \mu y$ one obtains

$$
d s^{2}=R^{2}\left[-\left(\rho^{2}-\frac{1}{\rho^{2}}\right) d \vec{t}^{2}+\rho^{2} d y_{i} d y_{i}+\frac{1}{\rho^{2}-\frac{1}{\rho^{2}}} d \rho^{2}+d \Omega_{5}^{2}\right] .
$$

The scale of the dimensionless gauge theory coordinates $\bar{t}, y_{i}$ in (44) is set by $R^{2} / \mu$ and one finds $f^{2}(\rho)=$ $\rho^{4}-1, g^{2}(\rho)=1$ and $\rho=1$ as the horizon location. The expressions for the dimensionless $q \bar{q}$ separation length (9) and potential energy (15) can be analytically computed [15, 16, 22]

$$
\begin{gathered}
\bar{L}\left(\rho_{0}\right)=\frac{(2 \pi)^{\frac{3}{2}}}{\Gamma\left[\frac{1}{4}\right]^{2}} \frac{\sqrt{\rho_{0}^{4}-1}}{\rho_{0}^{3}}{ }_{2} F_{1}\left(\frac{3}{4}, \frac{1}{2}, \frac{5}{4} ; \frac{1}{\rho_{0}^{4}}\right) \\
\bar{E}_{q \bar{q}}\left(\rho_{0}\right)=\frac{R^{2}}{\pi \alpha^{\prime}}\left[1-\frac{(2 \pi)^{\frac{3}{2}}}{2 \Gamma\left[\frac{1}{4}\right]^{2}} \rho_{0}{ }_{2} F_{1}\left(-\frac{1}{2},-\frac{1}{4}, \frac{1}{4} ; \frac{1}{\rho_{0}^{4}}\right)\right]
\end{gathered}
$$

Here $\rho_{0} \geq 1$ is the minimum radial position reached by the string and the minimum radial value $r_{\min }$ in (15) was taken to be the horizon location $r_{\min }=\mu$. One can easily check that in the small temperature limit $L T \ll 1$ (corresponding to $\rho_{0} \gg 1$ ) one recovers the zero temperature behavior (35)-(37). We have plotted in figures 4 and 6 the behavior of the length (45) and the energy (46) as functions of $\rho_{0}$. In figure 5 we plotted the relation $V_{\text {string }}(L)$ obtained from (45)-(46) by eliminating $\rho_{0}$, the result is a double valued function.

Figure 4 shows a maximum $\bar{L}_{c} \simeq 0.869$ at $\rho_{0 c} \simeq 1.177$ which implies that no smooth solution connecting the pair of quarks exists for $\bar{L}>\bar{L}_{c}$. The only existing solution for $\bar{L}>\bar{L}_{c}$ corresponds to two straight strings reaching the horizon. This configuration, used for the substraction in (15), is interpreted as the one corresponding to a pair of free quarks. The existence of a maximum in the $L\left(r_{0}\right)$ relation in $\mathrm{BH}$ backgrounds has been interpreted as the gravity dual of thermal bath screening [16]. Figure 4 also shows the existence of two branches of solutions for each string endpoints separation distance $L<L_{c}$. The left branch $\left(L^{\prime}>0\right)$ leads to a potential $V_{\text {string }}(L)$ not satisfying the conditions (17) and it should therefore be non-physical (upper curve in fig. 5). The rewarding result as we shall see is that string theory is wise: the left branch should not be trusted since it is unstable under small perturbations (see section 4.2).

The last point to comment is that although, as we shall show, all the $L^{\prime}<0$ region $\left(1.177<\rho_{0}<\infty\right)$ is stable under small perturbations, one expects the lower curve in figure 5 solution to be metastable whenever $E_{q \bar{q}}>0\left(0.754<\bar{L}<0.869\right.$ or $\left.1.177<\rho_{0}<1.524\right)$. The reason for this is that the (reference) two straight lines solution has $E_{q \bar{q}}=0$ and is therefore the absolute stable minimum fro $0.754<\bar{L}<0.869$ (see fig. 6). Taking this last fact into account [16] suggested to take $\bar{L}_{\max } \simeq 0.754$ as the screening length. 


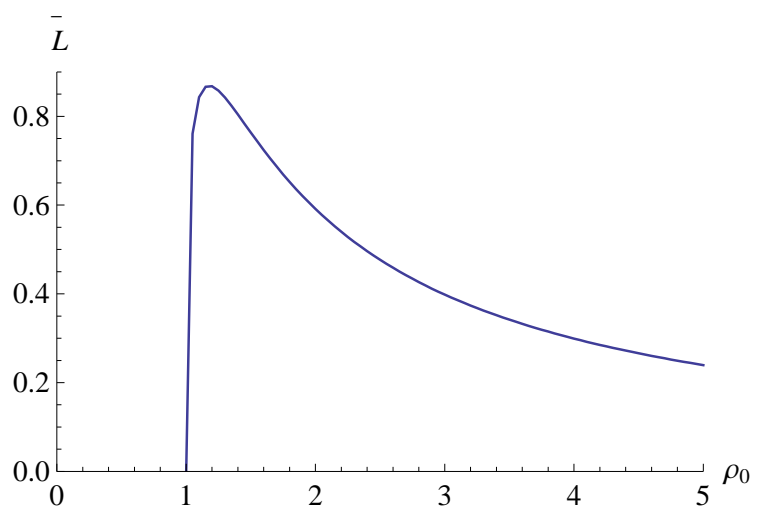

Figure 4: $L\left(\rho_{0}\right)$ relation (45): A maximum is observed for $\rho_{0} \simeq 1.177$. The two branches to the left/right of the maximum at $\rho_{0 c} \simeq 1.177$ lead to a double valued $V_{\text {string }}(L)$ relation in fig. 5 .

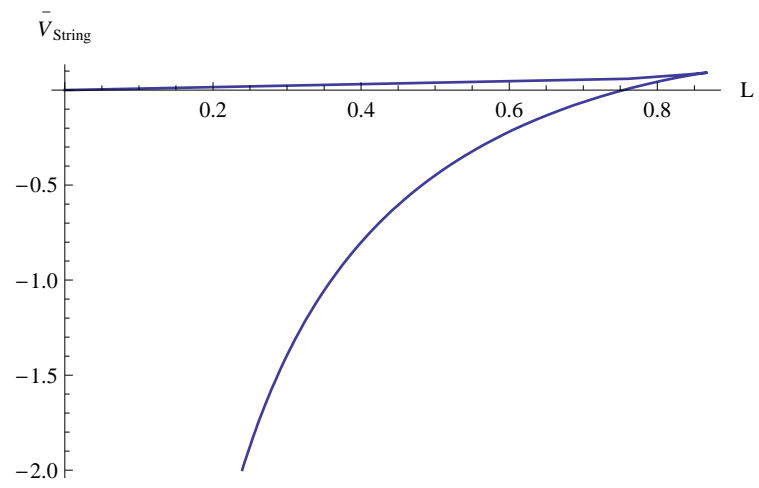

Figure 5: Double valued $V_{\text {string }}(L)$ obtained from (45) and (46) eliminating numerically $\rho_{0}$. The upper curve corresponding to the left branch in fig. 4 does not satisfy the conditions (17).

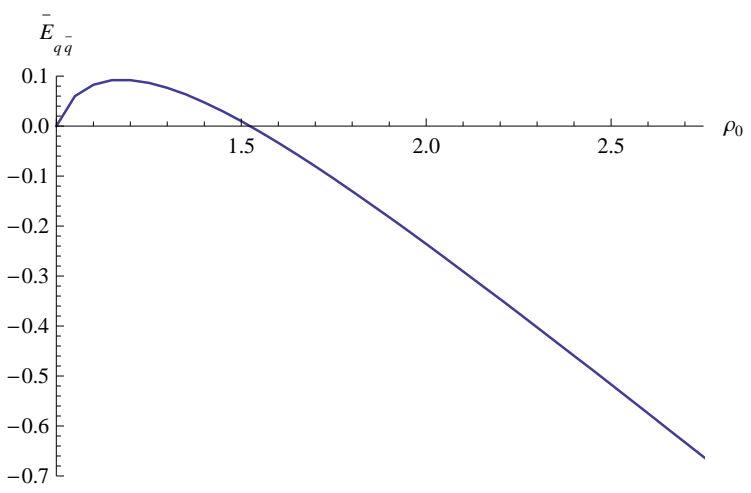

Figure 6: $\bar{E}_{q \bar{q}}\left(\rho_{0}\right)$ relation for thermal $\mathcal{N}=4 \mathrm{SYM}$. Eqn. (16) guarantees an extremum in $E_{q \bar{q}}\left(r_{0}\right)$ and $L\left(r_{0}\right)$ at the same value of $r_{0}$. The curve cuts the axis at $\rho_{0 m} \simeq 1.524$, for $\rho_{0}>\rho_{0 m}$ the Ushaped solution is an absolute minimum, for $\rho_{0}<\rho_{0 m}$ the two straight strings (reference) solution is the absolute minimum.

\subsection{Maldacena-Núñez background [25]}

The $r \approx 0$ of this background is supposed to describe qualitatively the IR regime of $d=4 \mathcal{N}=1 \mathrm{SYM}$ theory. The probe brane configuration leading to this solution consists of $N D 5$-branes wrapped on a finite 2 -cycle at the origin of the resolved conifold. When the backreaction of the $D 5$ on the geometry is taken into account, a transition flop occurs (see [30]) leading to a geometry with a smoothly collapsing $S^{2}$ and a finite $S^{3}$ at the origin as for the deformed conifold (see [31] for a review of the solution). The solution was independently found in the context of gauged supergravity in [32].

The metric can be written as 25]

$$
d s^{2}=\alpha^{\prime} N e^{\phi}\left[-d t^{2}+d x_{i} d x_{i}+d r^{2}+e^{2 h}\left(d \theta^{2}+\sin ^{2} \theta d \varphi^{2}\right)+\frac{1}{4}\left(w^{i}-A^{i}\right)^{2}\right],
$$

where $w^{i}(i=1,2,3)$ are the $s u(2)$ right-invariant forms

$$
w^{1}+i w^{2}=e^{-i \psi}(d \tilde{\theta}+i \sin \tilde{\theta} d \tilde{\varphi}), \quad w^{3}=d \psi+\cos \tilde{\theta} d \tilde{\varphi},
$$


and $A^{i}, \phi, h$ are given by

$$
\begin{aligned}
A^{1} & =-a(r) d \theta, \quad A^{2}=a(r) \sin \theta d \varphi, \quad A^{3}=-\cos \theta d \varphi \\
a(r) & =\frac{2 r}{\sinh 2 r} \\
e^{2 h} & =r \operatorname{coth} 2 r-\frac{r^{2}}{\sinh ^{2} 2 r}-\frac{1}{4} \\
e^{2 \phi} & =e^{2 \phi_{0}} \frac{\sinh 2 r}{2 e^{h}} .
\end{aligned}
$$

$\phi_{0}$ is an integration constant which sets the value of the dilaton $\phi$ at the origin (some authors write $g_{s}=e^{\phi_{0}}$ ). The $t, x_{i}, r$ coordinates are dimensionless and its scale is set by $\left(\alpha^{\prime} N\right)^{\frac{1}{2}}$. The metric (47) is regular in the $r \rightarrow 0$ limit with the 2-sphere $(\theta, \varphi)$ smoothly collapsing and the resulting topology of the spacetime is of the form $\mathcal{M}_{7} \times S^{3}$, contrary to the cases discussed before where the spacetimes were of the form $\mathcal{M}_{5} \times X^{5}$ with $X^{5}$ compact. The reason for this is that the background (47) models the $5+1$ gauge theory on the wrapped $D 5$-branes. Nevertheless, one expects to get an effective $3+1$ theory at energies $E<1 / R_{S^{2}}$, where $R_{S^{2}}$ is the radius of the sphere wrapped by the $D 5$.

The paradigmatic static U-shaped ansatz leads to $f^{2}(r)=g^{2}(r)=e^{2 \phi}$ and the separation length (9) and energy (15) take the form

$$
\begin{gathered}
\bar{L}\left(r_{0}\right)=2 \int_{r_{0}}^{\infty} \frac{e^{\phi\left(r_{0}\right)}}{\sqrt{e^{2 \phi(r)}-e^{2 \phi\left(r_{0}\right)}}} d r \\
\bar{E}_{q \bar{q}}\left(r_{0}\right)=\frac{N}{\pi}\left[\int_{r_{0}}^{\infty} \frac{e^{2 \phi(r)}}{\sqrt{e^{2 \phi(r)}-e^{2 \phi\left(r_{0}\right)}}} d r-\int_{0}^{\infty} e^{\phi(r)} d r\right] .
\end{gathered}
$$

The expression for the energy (51) can be rewritten in an illuminating form [8]

$$
\begin{aligned}
\bar{E}_{q \bar{q}}\left(r_{0}\right) & =\frac{N}{\pi}\left[\int_{r_{0}}^{\infty}\left(\frac{e^{2 \phi(r)}+e^{2 \phi\left(r_{0}\right)}-e^{2 \phi\left(r_{0}\right)}}{\sqrt{e^{2 \phi(r)}-e^{2 \phi\left(r_{0}\right)}}}-e^{\phi(r)}\right) d r-\int_{0}^{r_{0}} e^{\phi(r)} d r\right] \\
& =\frac{N}{\pi}\left[e^{\phi\left(r_{0}\right)} \frac{\bar{L}\left(r_{0}\right)}{2}+\int_{r_{0}}^{\infty} d r\left(\sqrt{e^{2 \phi(r)}-e^{2 \phi\left(r_{0}\right)}}-e^{\phi(r)}\right)-\int_{0}^{r_{0}} e^{\phi(r)} d r\right] .
\end{aligned}
$$

In the large $L$ limit the string reaches the bottom of the bulk $\left(r_{0} \rightarrow 0\right.$, see figure 7) and the last two terms in (52) do not contribute (see [8]). In the large $\bar{L} \gg 1$ limit we obtain reinserting units [25]

$$
V_{\text {string }}(L) \approx \frac{e^{\phi_{0}}}{2 \pi \alpha^{\prime}} L \quad \Rightarrow \quad T_{\text {string }}=\frac{e^{\phi_{0}}}{2 \pi \alpha^{\prime}} .
$$

The background (47) is therefore predicting linear confinement for large quarks separation in agreement with its proposed $d=4 \mathcal{N}=1 \mathrm{SYM}$ dual gauge theory. From the (chromoelectric) string tension (53) we see that the value of the dilaton at the origin $\phi_{0}$ relates to the dynamically generated scale of the dual gauge theory.

We plot in figures 7 and 8 the $\bar{L}\left(r_{0}\right)$ and $\bar{E}\left(r_{0}\right)$ relations (50) and (51). The divergence $L \rightarrow \infty$ at $r_{0} \rightarrow 0$ in fig. 7 is due to $\left.\frac{d}{d r}\left(e^{2 \phi(r)}\right)\right|_{r=0}=0$ (see [33] for a recent discussion).

The result for the potential $V_{\text {string }}(L)$ in figure 9 is rewarding, but the linear behavior occurs for configurations having energies above zero. A concern arises as to whether we should trust the result for $V_{\text {string }}>0$ (see last paragraph of section 3.2). The substraction in (51) corresponds to a pair of straight strings running along the radial direction with the remaining spatial coordinates kept fixed. Being the background regular, the strings cannot end at any point in the interior and the only possibility for a smooth reference solution is to place the string endpoints at antipodes on the $\varphi$ coordinate and having both the same $x_{1}$ coordinate (see the horizontal blue line in fig. 10) and the related discussion in sect. 3.1.2). We conclude that although the linear confinement occurs for configurations having $E_{q \bar{q}}>0$, the solution is stable and it cannot decay to the reference $E_{q \bar{q}}=0$ state since the two configurations being compared in (51) satisfy different boundary conditions. Figure 10 depicts the relevant worldsheets for the rectangular Wilson loop computations in smooth gravity backgrounds. 


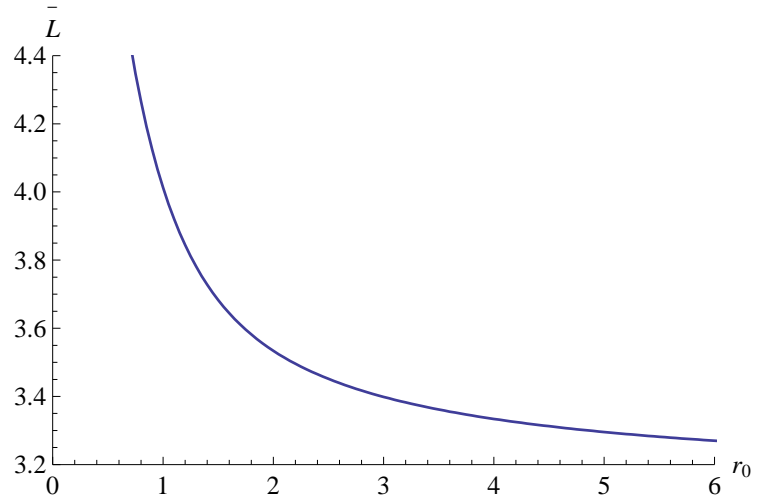

Figure 7: $\bar{L}\left(r_{0}\right)$ relation (50). MN solution.

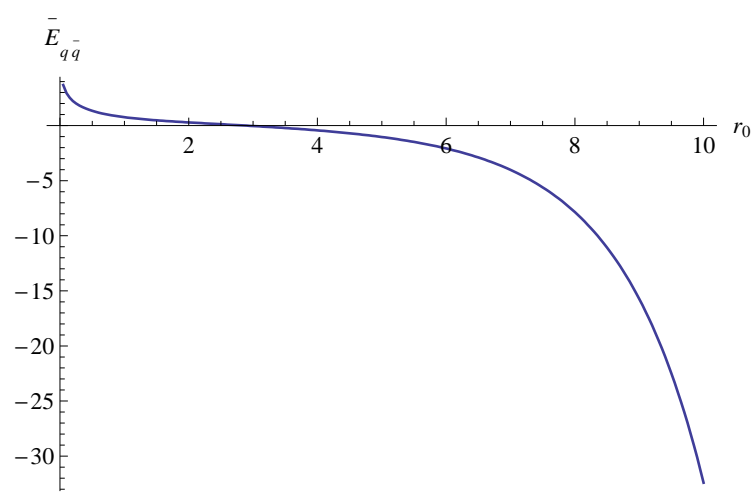

Figure 8: $E\left(r_{0}\right)$ relation (51). MN solution.

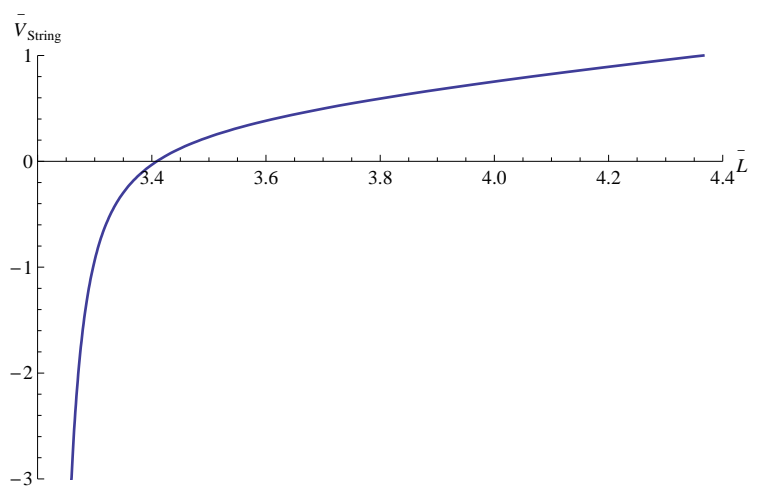

Figure 9: Potential energy $V_{\text {string }}(L)$ for the $q \bar{q}$ pair obtained for the Maldacena-Nuñez solution eliminating numerically $r_{0}$ from (50)-(51). Note the change of behavior from Coulomb like to linear.

\subsection{Klebanov-Strassler background [26]}

This background describes a $\mathcal{N}=1$ quiver gauge theory with bifundamental matter fields transforming under $S U(N+M) \times S U(N)$. The probe branes configuration leading to this geometry is constructed as: $N D 3$-branes in the apex of the singular conifold plus $M D 5$-branes wrapped in the topological $S^{2}$ of the conifold and sharing the remaining three dimensions with the $D 3$. The solution is supported by a constant dilaton $\phi=\phi_{0}$, which can be set to have $g_{s} \ll 1$ everywhere (contrary to the MN solution). The $\mathcal{N}=1$ flow $S U(N+M) \times S U(N) \rightarrow S U(M)$ realized through a cascade of Seiberg dualities in the gauge theory (see [34) manifests in the geometry in a varying 5 -form flux.

The metric reads [26]

$$
d s^{2}=g_{s} \alpha^{\prime} M\left[h^{-\frac{1}{2}}(r)\left(-d t^{2}+d x_{i} d x_{i}\right)+h^{\frac{1}{2}}(r) d s_{6}^{2}\right]
$$

The deformed conifold metric $d s_{6}$ can be written

$$
d s_{6}^{2}=\frac{1}{2} K(r)\left[\frac{\left(d r^{2}+\left(g^{5}\right)^{2}\right)}{3 K^{3}(r)}+\cosh ^{2} \frac{r}{2}\left(\left(g^{3}\right)^{2}+\left(g^{4}\right)^{2}\right)+\sinh ^{2} \frac{r}{2}\left(\left(g^{1}\right)^{2}+\left(g^{2}\right)^{2}\right)\right],
$$

where

$$
K(r)=\frac{[\sinh (2 r)-2 r]^{\frac{1}{3}}}{2^{\frac{1}{3}} \sinh r}
$$




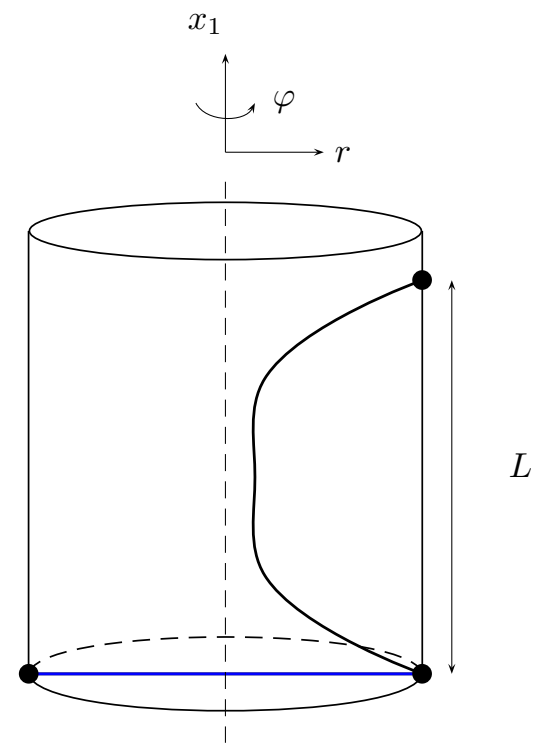

Figure 10: Geodesics employed for the computation of rectangular Wilson loops in smooth gravity backgrounds. The black curved vertical line depicts the U-shaped solution, the horizontal blue line is the smooth reference state with respect to which we compare the energy of the black line. The black and blue configurations satisfy different boundary conditions.

and the $g_{i}$ defined by

$$
g^{1}=\frac{e^{1}-e^{3}}{\sqrt{2}}, \quad g^{2}=\frac{e^{2}-e^{4}}{\sqrt{2}}, \quad g^{3}=\frac{e^{1}+e^{3}}{\sqrt{2}}, \quad g^{4}=\frac{e^{2}+e^{4}}{\sqrt{2}}, \quad g^{5}=e^{5},
$$

with

$$
\begin{gathered}
e^{1}=-\sin \theta_{1} d \phi_{1}, \quad e^{2}=d \theta_{1}, \quad e^{3}=-\sin \psi d \theta_{2}+\cos \psi \sin \theta_{2} d \phi_{2}, \\
e^{4}=\cos \psi d \theta_{2}+\sin \psi \sin \theta_{2} d \phi_{2}, \quad e^{5}=d \psi+\cos \theta_{1} d \phi_{1}+\cos \theta_{2} d \phi_{2} .
\end{gathered}
$$

The coordinates in (54) are dimesionless, the gauge theory coordinates $t, x_{i}$ scale is set by $\frac{g_{s} M \alpha^{\prime}}{\ell_{c f}}$, and the scale of the holographic coordinate $r$ is set by $\ell_{c f}$. The $h(r)$ factor takes the form

$$
h(r)=2^{\frac{2}{3}} \int_{r}^{\infty} d x \frac{x \operatorname{coth} x-1}{\sinh ^{2} x}(\sinh 2 x-2 x)^{\frac{1}{3}} .
$$

The background (54) is supported in part by a non-trivial $B_{\mu \nu}$ but the embedding we are considering gets no contribution from it [27]. The functions in (4) are given by $f^{2}(r)=\frac{1}{h(r)}, g^{2}(r)=\frac{1}{6 K^{2}(r)}$. The dimensionless expressions for the length (9) and the energy (15) are

$$
\begin{gathered}
\bar{L}\left(r_{0}\right)=2 \int_{r_{0}}^{\infty} \frac{d r}{\sqrt{6} K(r)} \frac{h(r)}{\sqrt{h\left(r_{0}\right)-h(r)}} . \\
\bar{E}_{q \bar{q}}\left(r_{0}\right)=\frac{g_{s} M}{\pi}\left[\int_{r_{0}}^{\infty} \frac{d r}{\sqrt{6} K(r)} \frac{\sqrt{h\left(r_{0}\right)}}{\sqrt{h\left(r_{0}\right)-h(r)}}-\int_{0}^{r_{0}} \frac{d r}{\sqrt{6} K(r)}\right] .
\end{gathered}
$$

In figures 11 and 12 we have plotted these two last expressions. As in the MN case a divergence is expected at $r_{0}=0$ since $\left.\frac{d h(r)}{d r}\right|_{r=0}=0$ Eliminating numerically $r_{0}$ from (60)-(61) we plot in figure 13 the $V_{\text {string }}(L)$ function. A linear relation for the interaction potential is observed for $L \gg 1$. Proceeding as in (52) we find the confining string tension to be

$$
T_{\text {string }}=\frac{1}{2 \pi \alpha^{\prime}} \frac{\ell_{c f}^{2}}{g_{s} \alpha^{\prime} M \sqrt{h_{0}}},
$$




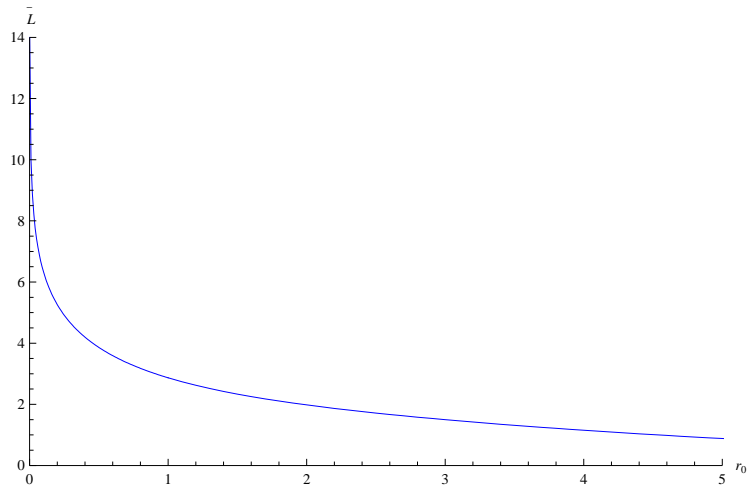

Figure 11: $\bar{L}\left(r_{0}\right)$ relation (60). KS solution.

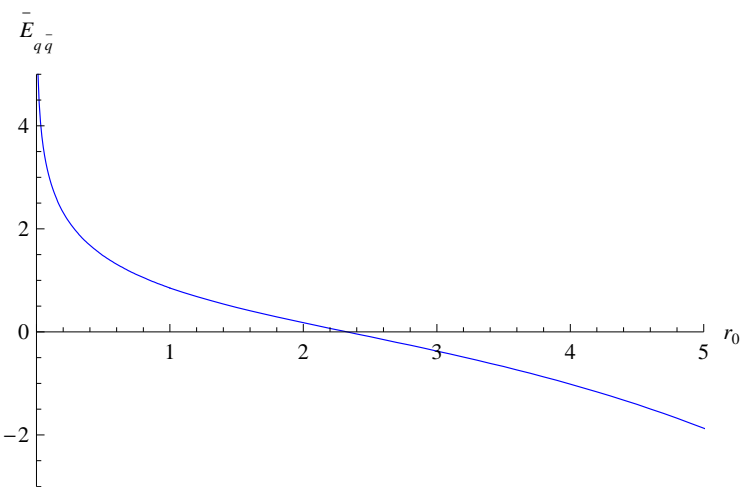

Figure 12: $\bar{E}_{q \bar{q}}\left(r_{0}\right)$ relation (61). KS solution.

where $h_{0}=h(0) \simeq 1.1398$. As in the Maldacena-Núñez case, the dominant contribution to the minimal area (1), in the large $L$ limit, comes from the $r \approx 0$ region. Again, a concern arises regarding whether one should trust the $E_{q \bar{q}}>0$ configurations, as discussed at the end of the last section and pictured in fig. 10 the $E_{q \bar{q}}>0$ embeddings are classically stable.

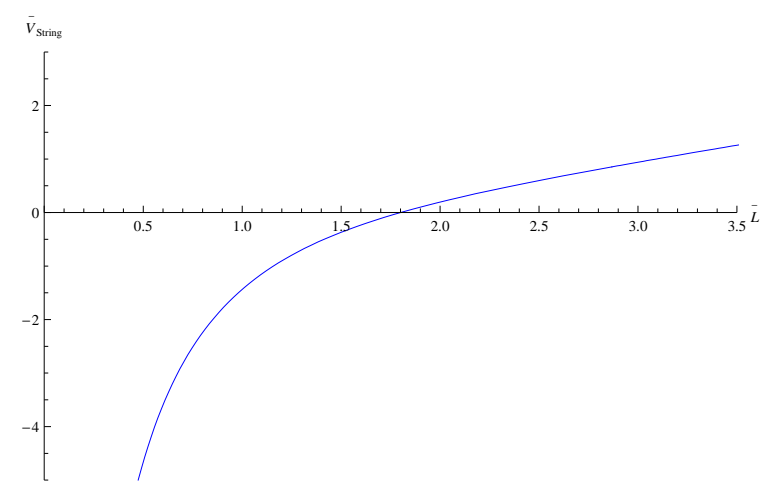

Figure 13: $V_{\text {string }}(L)$ relation for a rectangular Wilson loop in the KS solution.

\subsection{Generalized Maldacena-Núñez solutions [28],[29]}

This class of backgrounds was obtained in [28] generalizing the solution described in section 3.3. The solutions were thoroughly discussed in [29] an interpreted as dual to minimally supersymmetric gauge theories containing irrelevant dimension six operators. The operator drastically changes the UV behavior of the theories taking the solution 'away' from the near horizon of the D5-branes which generate the geometry. The analysis in [29] shows that the solutions asymptote, for large $r$, four dimensional Minkowski times the deformed conifold.

The generalized MN metric reads [28]

$$
\begin{aligned}
d s^{2}= & g_{s} \alpha^{\prime} N e^{4 f(r)}\left[-d t^{2}+d x_{i} d x_{i}+d r^{2}+e^{2 h(r)}\left(d \theta^{2}+\sin ^{2} \theta d \varphi^{2}\right)\right. \\
& \left.+\frac{e^{2 g(r)}}{4}\left(\left(w_{1}+a(r) d \theta\right)^{2}+\left(w_{2}-a(r) \sin \theta d \varphi\right)^{2}\right)+\frac{e^{2 k(r)}}{4}\left(w_{3}+\cos \theta d \varphi\right)^{2}\right] .
\end{aligned}
$$




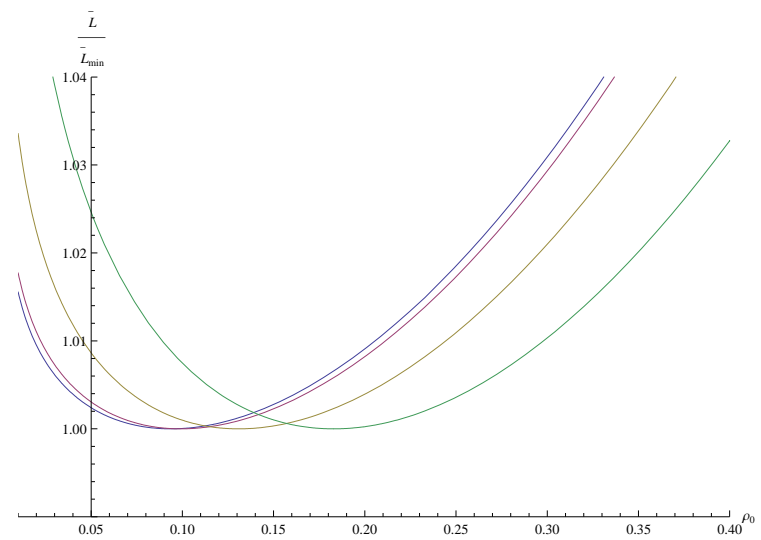

Figure 14: $L\left(\rho_{0}\right)$ relation (68). Generalized MN background with blue, violet, yellow and green curves corresponding to $\mu=$ $-1.8,-1.5,-1,-.8\left(\rho_{\infty}=7\right)$. A minimum quark separation length is observed.

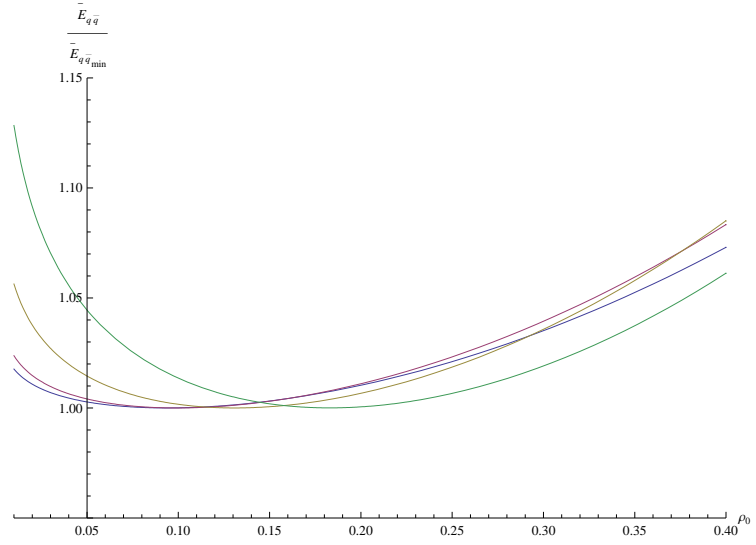

Figure 15: $E\left(r_{0}\right)$ relation (69). gMN background colors as in fig. 14

Making the change of coordinates $d \rho \equiv e^{-k(r)} d r$, the functions $a, k$ and $f$ satisfy

$$
\begin{aligned}
\partial_{\rho} a & =\frac{-2}{-1+2 \rho \operatorname{coth} 2 \rho}\left[e^{2 k} \frac{(a \cosh 2 \rho-1)^{2}}{\sinh 2 \rho}+a(2 \rho-a \sinh 2 \rho)\right] \\
\partial_{\rho} k & =\frac{2\left(1+a^{2}-2 a \cosh 2 \rho\right)^{-1}}{-1+2 \rho \operatorname{coth} 2 \rho}\left[e^{2 k} a \sinh 2 \rho(a \cosh 2 \rho-1)+\left(2 \rho-4 a \rho \cosh 2 \rho+\frac{a^{2}}{2} \sinh 4 \rho\right)\right] \\
\partial_{\rho} f & =-\frac{1}{4 \sinh ^{2} 2 \rho}\left[\frac{(1-a \cosh 2 \rho)^{2}(-4 \rho+\sinh 4 \rho)}{\left(1+a^{2}-2 a \cosh 2 \rho\right)(-1+2 \rho \operatorname{coth} 2 \rho)}\right]
\end{aligned}
$$

and the $g(\rho), h(\rho)$ functions in (63) are given by

$$
e^{2 g}=\frac{b \cosh 2 \rho-1}{a \cosh 2 \rho-1}, \quad e^{2 h}=\frac{e^{2 g}}{4}\left(2 a \cosh 2 \rho-1-a^{2}\right), \quad \text { with } \quad b(\rho)=\frac{2 \rho}{\sinh 2 \rho} .
$$

The first two differential equations in (64) have a one parameter family of regular solutions. For small $r$ one finds [28]

$$
a(\rho)=1+\mu \rho^{2}+\ldots, \quad e^{2 k(\rho)}=\frac{4}{6+3 \mu}-\frac{20+36 \mu+9 \mu^{2}}{15(2+\mu)} \rho^{2}+\ldots
$$

with $\mu$ taking values in the interval $\left(-2,-\frac{2}{3}\right)$. Inserting (66) into the third equation of (64) and into (65) one obtains

$$
e^{2 g(\rho)}=\frac{4}{6+3 \mu}+\ldots, \quad e^{2 h(\rho)}=\frac{4 \rho^{2}}{6+3 \mu}+\ldots, \quad e^{2 f(\rho)}=1+\frac{(2+\mu)^{2}}{8} \rho^{2}+\ldots
$$

The arbitrary constant for $f$ following from (64) was factored out as $g_{s}$ in (63). The limit values for $\mu$ give known solutions: the $\mu=-\frac{2}{3}$ case reproduces the MN solution of section 3.3 with $\phi=4 f(k=$ const.), and the case $\mu=-2$ case leads to 4 -dimensional Minkowski space times the deformed conifold ( $\phi$ being constant in this case). Finally, the $\rho \rightarrow \infty$ limit of all solutions (except $\mu=-\frac{2}{3}$ ) asymptotes the deformed conifold metric (see [28] for details).

The length (9) and energy (15) expressions are given by

$$
\bar{L}\left(\rho_{0}\right)=2 \int_{\rho_{0}}^{\rho_{\infty}} \frac{e^{4 f\left(\rho_{0}\right)}}{\sqrt{e^{8 f(\rho)}-e^{8 f\left(\rho_{0}\right)}}} e^{k(\rho)} d \rho
$$




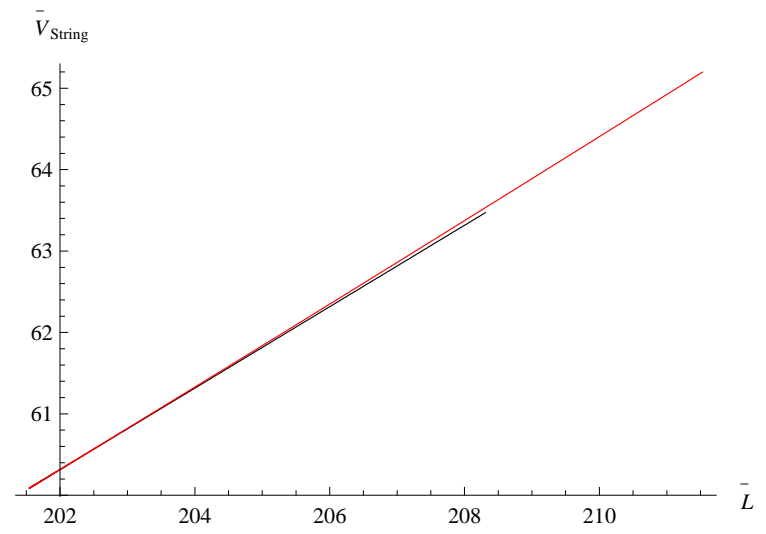

Figure 16: Double valued $V_{\text {string }}(L)$ relation for gMN background with $\mu=-1$. The upper red curve is the unphysical branch corresponding to string embeddings to the right of the minimum in figure 14. The curve does not reach the origin, manifesting a minimum quark sep-

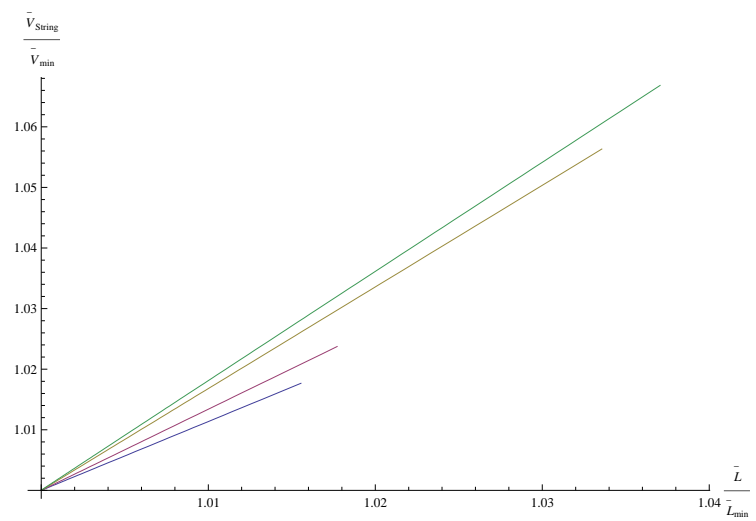

Figure 17: Normalized linear $\bar{V}_{\text {string }}(L)$ relation for the physical (left) branches of figure 14. Colors as in fig. 14. aration length.

$$
\bar{E}_{q \bar{q}}\left(\rho_{0}\right)=\frac{N g_{s}}{\pi}\left[\int_{\rho_{0}}^{\rho_{\infty}} \frac{e^{8 f(\rho)}}{\sqrt{e^{8 f(\rho)}-e^{8 f\left(\rho_{0}\right)}}} e^{k(\rho)} d \rho-\int_{0}^{\rho_{\infty}} e^{4 f(\rho)} e^{k(\rho)} d \rho\right] .
$$

Note that the radial integration in both expressions extends up to a finite distance $\rho_{\infty}$. The reason, noted in [28], being that (68) is UV divergent 8 . We should therefore have in mind that the string computation corresponds to probing the dual gauge theory with very massive for $\rho_{\infty} \gg 1$ (but not infinite mass) quarks. Moreover since the string endpoints are fixed at a finite radial distance it can be checked at the probe string does not reach the gauge theory brane along a normal direction.

In figures 14 and 15 we plot numerical solutions of (68) and (69) for various values of $\mu$. Figure (14) shows that $L\left(\rho_{0}\right)$ attains a global minimum for all values of $\mu$ (except $\mu \neq-\frac{2}{3}$ ). In other words, no solution exists for quarks separations $L<L_{\text {min }}$. It is interesting to note that the minimum value for $L$ is attained, for all values of $\mu$, in a rather small region in the $\rho$ coordinate near the origin. Based on the concavity considerations discussed at the end of section 2.1 we expect the string worldsheets to the left of the minimum $\rho_{c}$ to be physically meaningful (stable) and the ones to the right of $\rho_{c}$ to be unphysical (unstable). We will show in the following section that this is in fact the case by analyzing quadratic fluctuations around the solutions. Negative eigenvalues appear for the $L^{\prime}\left(\rho_{0}\right)>0$ branch of solutions.

We finally plot in figures (16) and (17) the $V_{\text {string }}(L)$ relation for the generalized MN backgrounds where a linear confining behavior is observed. Figure (16) shows the double valued $V_{\text {string }}(L)$ relation for $\mu=-1$, the upper red branch (unphysical) corresponds to the string configurations to the right of the minimum in fig. (14). In figure 17 we show the $V_{\text {string }}(L)$ relation for the physical branches of figure 14 for several values of $\mu$. Proceeding as in (52) one finds that all solutions lead to a $\mu$-independent string tension

$$
T_{\text {string }}=\frac{g_{s}}{2 \pi \alpha^{\prime}} .
$$

\section{Stability Analysis}

In this section we study, for the backgrounds presented in the previous section, the eigenvalue problem given by the equation of motion (22) for in-plane fluctuations in the $r$-gauge. We are interested in searching for unstable modes. For the reasons discussed at the end of section 2.1 our aim is to show that negative

\footnotetext{
${ }^{8}$ See $\left[33\right.$ for a discussion on divergences when computing the length function $L\left(r_{0}\right)$.
} 
eigenvalues $\left(\omega^{2}<0\right)$ appear for string configurations belonging to regions where $L^{\prime}\left(r_{0}\right)>0$. We study even solutions, this means that we choose $C_{1}^{\prime}=1$ and $C_{0}^{\prime}=0$ in (25) as the initial condition at the tip 9 . We numerically implement this conditions as (see (31))

$$
\begin{aligned}
\delta x_{1}(r)+2\left(r-r_{0}\right) \frac{d \delta x_{1}(r)}{d r} & =0, \quad r \rightarrow r_{0} \\
\sqrt{r-r_{0}} \delta x_{1}(r) & =1, \quad r \rightarrow r_{0} .
\end{aligned}
$$

Solving numerically, the allowed eigenvalues $\omega^{2}$ for (22) are obtained by demanding $\delta x_{1}(r)$ to be a normalizable solution

$$
\delta x_{1}(r)=0, \quad r \rightarrow \infty .
$$

For completeness we recall now the relation between zero modes for in-plane modes and critical points of the $L\left(r_{0}\right)$ function [15]. The zero mode solution of (22) can be immediately written down

$$
\delta x_{1}^{(0)}(r)=C \int_{r}^{\infty} d \bar{r} \frac{g(\bar{r}) f(\bar{r})}{\left(f^{2}(\bar{r})-f^{2}\left(r_{0}\right)\right)^{\frac{3}{2}}}+C^{\prime}
$$

where $C^{\prime}, C$ are integration constants, $C^{\prime}=0$ to get a normalizable solution and we set $C^{\prime}=1$. Integrating by parts in (73) and using (10) one obtains

$$
\begin{aligned}
\delta x_{1}^{(0)}(r) & =-\int_{r}^{\infty} d \bar{r} \frac{g(\bar{r})}{f^{\prime}(\bar{r})} \frac{d}{d \bar{r}}\left(\frac{1}{\sqrt{f^{2}(\bar{r})-f^{2}\left(r_{0}\right)}}\right) \\
& =\frac{g(r)}{f^{\prime}(r) \sqrt{f^{2}(r)-f^{2}\left(r_{0}\right)}}+\frac{L^{\prime}(r)}{2 f^{\prime}(r)} .
\end{aligned}
$$

Expanding this last expression around the tip $r=r_{0}$ one has

$$
\delta x_{1}^{(0)}(r)=\frac{g\left(r_{0}\right)}{\sqrt{2}\left(f^{\prime}\left(r_{0}\right)\right)^{\frac{3}{2}}} \frac{1}{\sqrt{r-r_{0}}}+\frac{L^{\prime}\left(r_{0}\right)}{2 f^{\prime}\left(r_{0}\right)}+\mathcal{O}\left(\sqrt{r-r_{0}}\right) .
$$

Generically the first factor in the rhs of (75) is non-zero, so a necessary and sufficient condition for obtaining an even zero mode solution (see (71) ) requires the second term in (75) to cancel, equivalently $r_{0}$ must be a critical point of the $L\left(r_{0}\right)$ length function [15].

\section{1 $A d S_{5}$}

The in-plane fluctuations equation of motion (22) for the $A d S$ spacetime in Poincare coordinates (33) takes the form

$$
\left[\frac{d}{d r}\left(\frac{\left(r^{4}-r_{0}^{4}\right)^{\frac{3}{2}}}{r^{2}} \frac{d}{d r}\right)+\omega^{2} R^{4} \frac{\sqrt{r^{4}-r_{0}^{4}}}{r^{2}}\right] \delta x_{1}(r)=0 \quad 0<r_{0} \leq r<\infty .
$$

Dilatation invariance implies that one should be able to factor out the $r_{0}$ dependence. Making $r=r_{0} \rho$ one obtains

$$
\left[\frac{d}{d \rho}\left(\frac{\left(\rho^{4}-1\right)^{\frac{3}{2}}}{\rho^{2}} \frac{d}{d \rho}\right)+\frac{\omega^{2} R^{4}}{r_{0}^{2}} \frac{\sqrt{\rho^{4}-1}}{\rho^{2}}\right] \delta x_{1}(\rho)=0 .
$$

The asymptotic behavior $(\rho \rightarrow \infty)$ of $(77)$ reads

$$
\left[\frac{d}{d \rho}\left(\rho^{4} \frac{d}{d \rho}\right)+\frac{\omega^{2} R^{4}}{r_{0}^{2}}\right] \delta x_{1}(\rho) \approx 0, \quad \rho \gg 1,
$$

whose solutions are

$$
\delta x_{1}(\rho) \approx \alpha_{0}+\frac{\alpha_{1}}{\rho^{3}}, \quad \rho \gg 1,
$$

\footnotetext{
${ }^{9}$ Even solutions correspond to arbitrary $C_{1}^{\prime}$ at the tip, its value fixes the normalization of the solution.
} 


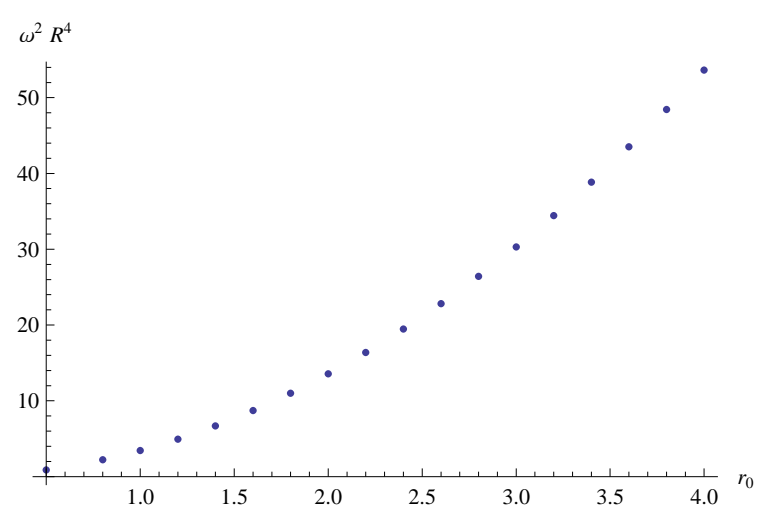

Figure 18: Lowest numerical eigenvalue $\omega^{2}$ of (76) giving a normalizable solution as a function of $r_{0}$.

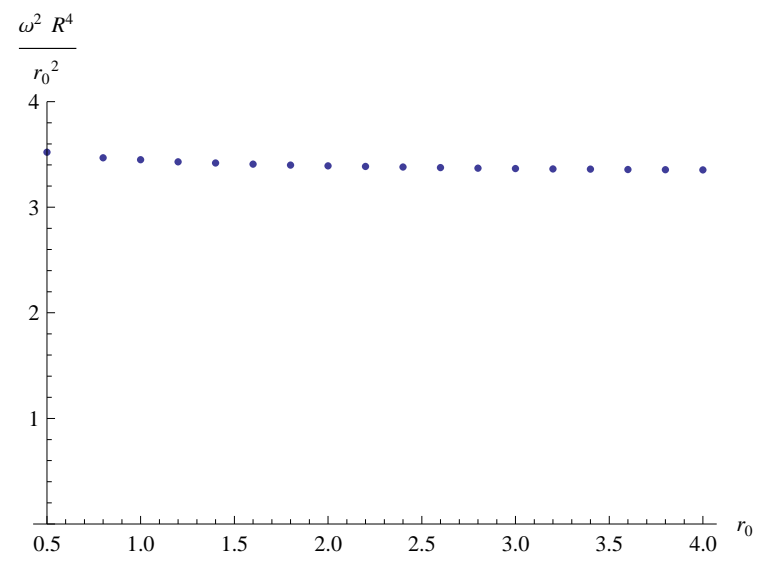

Figure 19: Properly normalized lowest eigenvalues of fig. 18 as a function of $r_{0}$ (see (77)).

with $\alpha_{0}, \alpha_{1}$ integration constants. The behavior (79) implies that normalizable solutions $\left(\alpha_{0}=0\right)$ will be found only for particular (discrete) values $\omega_{n}^{2}$.

As a test for our shooting method, we have numerically integrated (76) for different values of $r_{0}$ and determined the minimum $\omega^{2}$ eigenvalues leading to a normalizable solution. In figure 18 we plot these $\omega^{2}$ as a function of $r_{0}$. They are positive for all values of $r_{0}$, signaling the stability of the U-shaped string configuration. In fig. 19 we show the expected $r_{0}$-independence of the mode when properly normalized (see (77)). In the following table we show the first eigenvalues corresponding to even boundary conditions at the tip.

\begin{tabular}{|c||c|}
\hline & $\omega_{n}^{2} R^{4} / r_{0}^{2}$ \\
\hline$n=1$ & 3.450 \\
$n=3$ & 22.113 \\
$n=5$ & 52.325 \\
$n=7$ & 94.558 \\
$n=9$ & 148.845 \\
\hline
\end{tabular}

In section 5 we prove the stability of the configuration by transforming the differential equation (76) into a Schrodinger like one (see appendix $\underline{\mathrm{A}}$ ).

\subsection{Non-Extremal D3-branes}

The in-plane fluctuation equation of motion (22) for the background (43) takes the form

$$
\left[\frac{d}{d \rho}\left(\frac{\left(\rho^{4}-\rho_{0}^{4}\right)^{\frac{3}{2}}}{\sqrt{\rho^{4}-1}} \frac{d}{d \rho}\right)+\frac{\omega^{2} R^{4}}{\mu^{2}} \frac{\rho^{4} \sqrt{\rho^{4}-\rho_{0}^{4}}}{\left(\rho^{4}-1\right)^{\frac{3}{2}}}\right] \delta x_{1}(\rho)=0, \quad 1<\rho_{0} \leq \rho<\infty .
$$

The background (43) asymptotes $A d S$ and therefore the asymptotic $(\rho \rightarrow \infty)$ behavior of the solutions of (80) is given by (79). As in the case of the last subsection, we expect to find a discrete set of eigenvalues leading to normalizable solutions.

We plot in figure 20 the lowest eigenvalue we found when numerically solving (80) looking for normalizabe solutions. A zero mode appears precisely at the critical point of the length function $L\left(\rho_{0}\right)$, that is for $\rho_{0} \simeq 1.177$ (see fig. 4), in agreement with (75).

We conclude that the (left) branch in figure 4 having $L^{\prime}\left(\rho_{0}\right)>0$ is unstable under linear perturbations. Finally, note that the numerical analysis indicates that solutions on the (right) branch in figure 4 having $L^{\prime}\left(\rho_{0}\right)<0$ are stable under linear perturbations. Nevertheless, as discussed at the end of section 3.2 one expects the solutions with $1.177<\rho_{0}<1.524$ to be metastable, decaying to a pair of free quarks. 


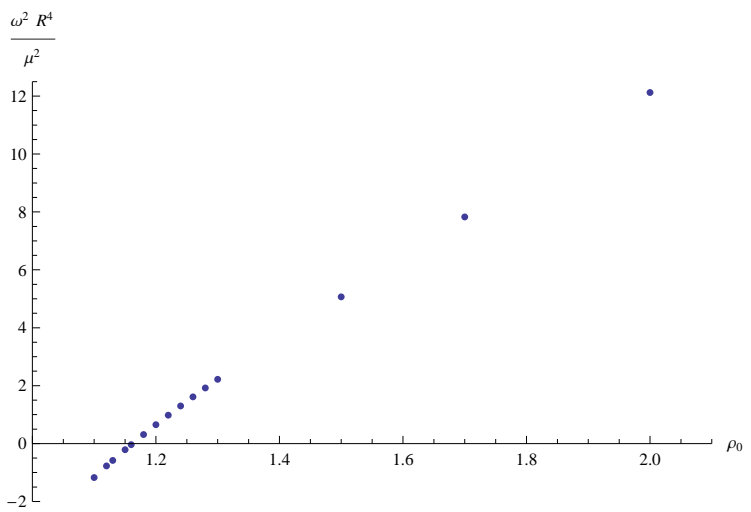

Figure 20: Lowest $\omega^{2}$ eigenvalue of (80) giving a normalizable solution as a function of $\rho_{0}$. A zero mode appears for $\rho_{0} \simeq 1.177$. The classical solutions with $\rho_{0}<1.177$ are unstable against linear perturbations.

\subsection{Maldacena-Núñez background}

The in-plane fluctuation equation of motion for the Maldacena-Núñez background (47) takes the following form (22)

$$
\left[\frac{d}{d r}\left(\frac{\left(e^{2 \phi(r)}-e^{2 \phi\left(r_{0}\right)}\right)^{\frac{3}{2}}}{e^{2 \phi(r)}} \frac{d}{d r}\right)+\bar{\omega}^{2} \sqrt{e^{2 \phi(r)}-e^{2 \phi\left(r_{0}\right)}}\right] \delta x_{1}(r)=0, \quad 0<r_{0} \leq r<\infty .
$$

where $\bar{\omega}^{2}=\omega^{2} \alpha^{\prime} N$. We now compute the asymptotic behavior of (181) to see whether we should expect a quantized spectrum or not. In the $r \rightarrow \infty$ limit the equation of motion for $\delta x_{1}(r)$ reads

$$
\left[\frac{d}{d r}\left(e^{r} r^{-\frac{1}{4}} \frac{d}{d r}\right)+\bar{\omega}^{2} e^{r} r^{-\frac{1}{4}}\right] \delta x_{1}(r)=0, \quad r \gg 1,
$$

where we used that $e^{2 \phi(r)} \rightarrow e^{2 r} r^{-\frac{1}{2}}$ for $r \gg 1$. This last equation can be written as

$$
\left[\frac{d^{2}}{d r^{2}}+\left(1-\frac{1}{4 r}\right) \frac{d}{d r}+\bar{\omega}^{2}\right] \delta x_{1}(r)=0, \quad r \gg 1 .
$$

The $r^{-1}$ term can be omitted in the large $r$ limit and the asymptotic solution to (81) is then

$$
\delta x_{1}(r) \simeq e^{-\frac{1}{2} r}\left(\beta_{0} e^{r \alpha}+\beta_{1} e^{-r \alpha}\right), \quad r \gg 1,
$$

where $\alpha=\frac{\sqrt{1-4 \bar{\omega}^{2}}}{2}$. From (83) it follows that any $\bar{\omega}^{2}>0$ lead to normalizable solutions, the spectrum of stable in-plane fluctuation is therefore continuum. In the $\bar{\omega}^{2} \leq 0$ case $\left(\alpha \geq \frac{1}{2}\right) \beta_{0}$ must be set to zero and we have the possibility of getting a discrete spectrum of negative eigenvalues. Our numerical analysis could not find any normalizable negative eigenmodes, suggesting the stability of the classical configuration in agreement with the concavity condition (17).

In the section 5 we show that negative eigenvalues does not exist from the study of a Schrodinger equation analysis of (81) (see appendix A).

\subsection{Klebanov-Strassler background}

The equation of motion for the in-plane fluctuation in this case takes the form

$$
\left[\frac{d}{d r}\left(\frac{K(r)}{h(r)}\left(1-\frac{h(r)}{h\left(r_{0}\right)}\right)^{\frac{3}{2}} \frac{d}{d r}\right)+\bar{\omega}^{2} \frac{1}{6 K(r)} \sqrt{1-\frac{h(r)}{h\left(r_{0}\right)}}\right] \delta x_{1}(r)=0, \quad 0<r_{0} \leq r<\infty,
$$


with $\bar{\omega}^{2}$ dimensionless and $K(r)$ and $h(r)$ given by (56) and (59) respectively. From the $r \rightarrow \infty$ limit of $K(r)$ and $h(r)$ one obtains

$$
\left[\frac{d}{d r}\left(\frac{e^{r}}{r} \frac{d}{d r}\right)+\bar{\omega}^{2} \frac{e^{\frac{r}{3}}}{2^{\frac{4}{3}}}\right] \delta x_{1}(r)=0, \quad r \gg 1
$$

which gives

$$
\left[\frac{d^{2}}{d r^{2}}+\left(1-\frac{1}{r}\right) \frac{d}{d r}+\bar{\omega}^{2} \frac{r e^{-\frac{2}{3} r}}{2^{\frac{4}{3}}}\right] \delta x_{1}(r)=0, \quad r \gg 1 .
$$

In the large $r$ limit the $r^{-1}$ and the last term in (86) can be omitted and the in-plane fluctuation asymptotics turns to be

$$
\delta x_{1}(r) \simeq \alpha_{0}+\alpha_{1} e^{-r}, \quad r \gg 1 .
$$

The integration constant $\alpha_{0}$ must be set to zero to obtain normalizable solutions and we therefore expect to get a discrete eigenvalue spectrum. In figure (21) we plot the lowest eigenvalue of (84) we have found

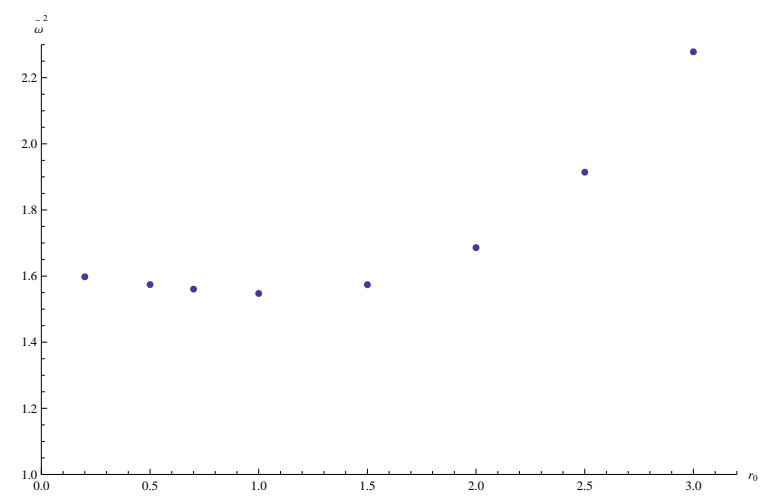

Figure 21: Numerical solution for the lowest $\omega^{2}$ leading to a normalizable solution as a function of $r_{0}$ for the Klebanov-Strassler background. No negative eigenvalues where found.

numerically leading to a normalizable solution as a function of $r_{0}$. We have not found numerically any negative eigenvalues. In section 5 we will prove the stability of the classical solution transforming (84) to a Schrodinger like equation and showing that no negative modes can appear (see appendix A).

\subsection{Generalized Maldacena-Núñez}

The equation of motion for the in-plane fluctuation $\delta x_{1}(\rho)$ in the backgrounds (63) takes the form

$$
\left[\frac{d}{d \rho}\left(\frac{\left(e^{8 f(\rho)}-e^{8 f\left(\rho_{0}\right)}\right)^{\frac{3}{2}}}{e^{8 f(\rho)+k(\rho)}} \frac{d}{d \rho}\right)+\bar{\omega}^{2} e^{k(\rho)} \sqrt{e^{8 f(\rho)}-e^{8 f\left(\rho_{0}\right)}}\right] \delta x_{1}(\rho)=0, \quad 0<\rho_{0} \leq \rho<\infty .
$$

In the $\mu=-\frac{2}{3}$ case $(k(\rho)=$ const. $)$ the equation (81) for the Maldacena-Nuñez background is recovered (from now on we consider $\mu \neq-\frac{2}{3}$ ). In the large $\rho$ limit the gMN solutions asymptote the deformed conifold and the $f$ function approaches a constant $f_{\infty}$, the asymptotic behavior is then given by

$$
\left[e^{-k(\rho)} \frac{d}{d \rho}\left(e^{-k(\rho)} \frac{d}{d \rho}\right)+\bar{\omega}^{2} \frac{e^{8 f_{\infty}}}{e^{8 f_{\infty}}-e^{8 f\left(\rho_{0}\right)}}\right] \delta x_{1}(\rho)=0, \quad \rho \gg 1 .
$$

Returning to the original $r$ variable in (지) $\left(d r=e^{k(\rho)} d \rho\right)$ one obtains

$$
\left[\frac{d^{2}}{d r^{2}}+\tilde{\omega}^{2}\right] \delta x_{1}(r)=0, \quad r \gg 1
$$




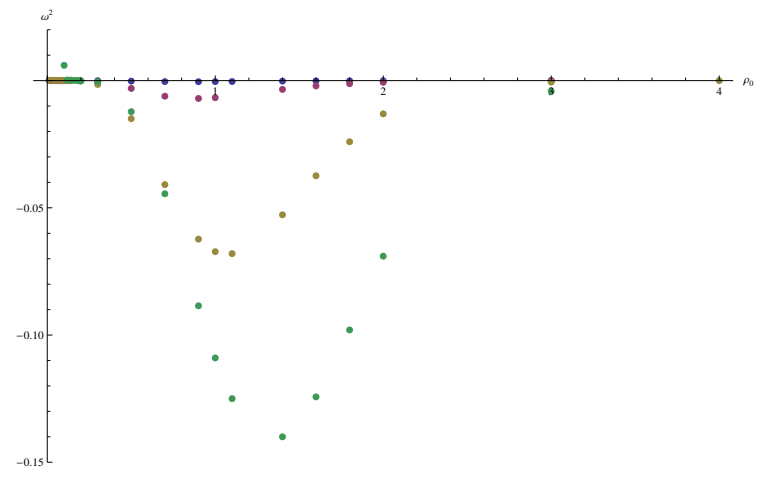

Figure 22: Lowest $\omega^{2}$ eigenvalue of (88) leading to a normalizable solution as a function of $\rho_{0}$. Negative (unstable) modes are found precisely for the classical embeddings satisfying $L^{\prime}\left(\rho_{0}\right)>0$. Colors as in fig. 14.

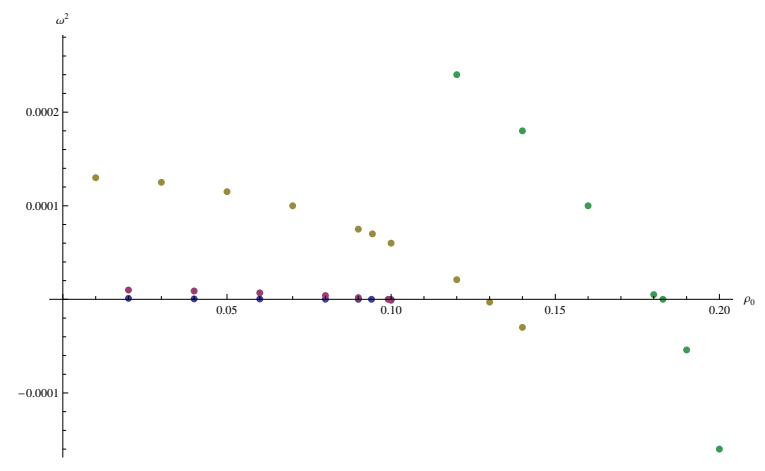

Figure 23: Zoom of figure 22 near the origin. The lowest eigenvalues are positive for $L^{\prime}\left(\rho_{0}\right)<0$ solutions. A zero mode appears precisely for the critical values of the length function $L\left(\rho_{0}\right)$ (see fig. 14).

whose solutions are plane waves $e^{ \pm i \tilde{\omega} r}$ for $\bar{\omega}^{2}>0$ and real exponentials $e^{ \pm \tilde{\omega} r}$ for $\bar{\omega}^{2}<0$ case. We conclude that no normalizable solutions exist for $\bar{\omega}^{2}>0$. A word of caution, as discussed in section 3.5 the gauge theory brane must be placed at a finite distance $\rho_{\infty}$, therefore for (88) defined on $\rho_{0} \leq \rho \leq \rho_{\infty}$ positive eigenvalues will exist. In the $\bar{\omega}^{2}<0$ case the possibility for negative eigenmodes exists and in fact we find normalizable negative mode solutions precisely for the classical solutions region where the convexity condition (17) is not satisfied. In the figures 22 and 23 we plot the minimal eigenvalues leading to normalizable solutions we found numerically as a function of $r_{0}$. We found complete agreement with figure 14: no instabilities are found for classical solutions satisfying $L^{\prime}\left(r_{0}\right)<0$, on the other hand, we find negative (unstable) modes for the right branch curve $\left(L^{\prime}\left(r_{0}\right)>0\right.$ solutions) in figure 14. These results are gratifying since unstable modes are found precisely for the classical embeddings which do not satisfy the conditions (17). In the following section we review this results by transforming the equation into a Schrodinger like problem.

\section{Schrodinger Potentials Analysis}

In this section we analyze the fluctuation equation of motion (22) transforming it to a Schrodinger like equation (see appendix $\mathrm{A}$ ). From the form of the potential it is possible in some cases to show that no negative eigenvalues can appear and therefore to prove the stability of the corresponding classical embeddings.

\section{$5.1 \quad A d S_{5}$}

The Schrodinger potential (107) for the equation (77) takes form [15]

$$
V(\rho)=2 \frac{\rho^{4}-1}{\rho^{2}}, \quad \rho \in[1, \infty),
$$

here $\rho$ should be understood as $\rho=\rho(y)$. The change of variables (105) leading to the Schrodinger equation (106) can be analytically computed

$$
y(\rho)=y_{0}-\frac{1}{4} \mathrm{~B}\left(\frac{1}{\rho^{4}} ; \frac{1}{4}, \frac{1}{2}\right),
$$

with $y_{0}=\frac{\Gamma\left[\frac{1}{4}\right]^{2}}{4 \sqrt{2 \pi}}$. The half line $\rho \in[1, \infty)$ of the original Sturm-Liouville problem, under the change of variables (92), maps to the finite interval $y \in\left[0, y_{0}\right]$, the potential (91) diverging at $y_{0}$. We have therefore obtained a Schrodinger problem defined on a finite interval with canonical boundary conditions (see (108)(109)) hence a discrete spectrum will result, moreover, since the potential (91) is positive definite a standard 


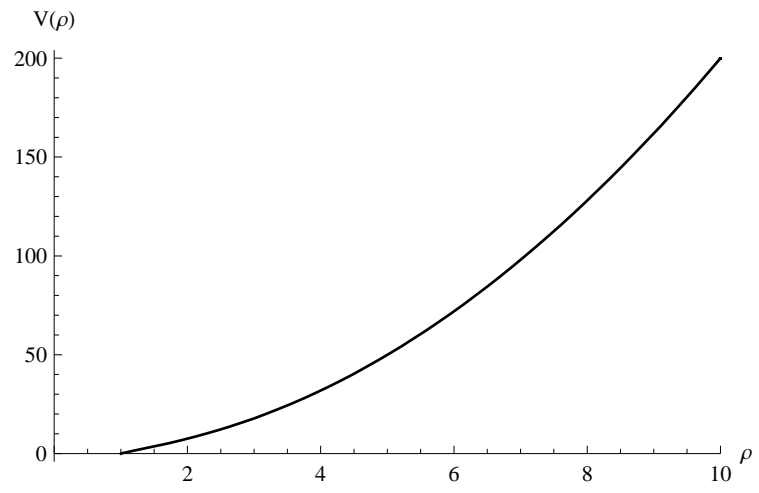

Figure 24: Schrodinger potential (91) for inplane fluctuations in $A d S$ the positive definite property of it guarantees that no negative eigenmodes exist.

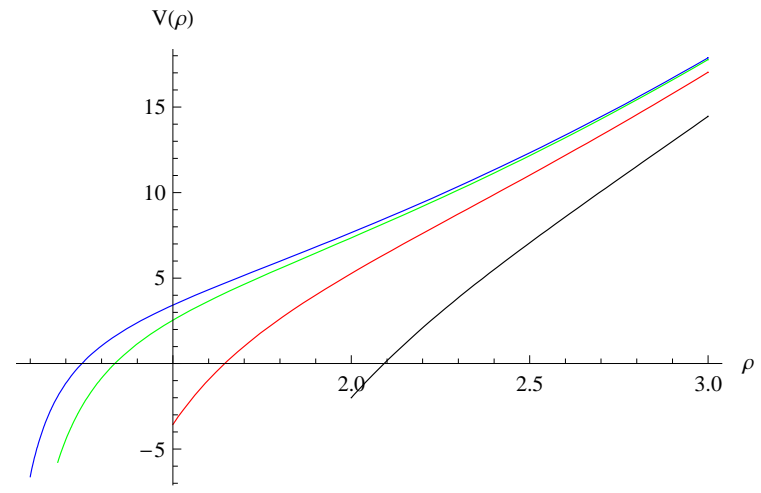

Figure 25: $V\left(\rho, \rho_{0}\right)$ (eqn.(93)) for different $\rho_{0}$ values. The blue line corresponds to $\rho_{0}=1.1$, the green to the critical value $\rho_{0}=1.177$ and the red, black lines to $\rho_{0}=1,2$. The region where the potential is negative diminishes as $\rho_{0}$ increases. Negative eigenmodes cease to exist for $\rho_{0} \geq 1.177$

QM argument tell us that no negative eigenvalue solutions exist. We conclude that the $A d S$ embedding given by (34) is stable under linear perturbations. Figure 24 shows a plot of the potential (91) as a function of $\rho$, the true variable for the Schrodinger problem is $y$ given by (92) and it amounts to a rescaling of the horizontal axis in fig. 24 mapping $\rho=\infty$ to a finite distance.

\section{$5.2 A d S_{5}$-Schwarzschild}

The Schrodinger potential (107) for the in-plane fluctuation equation (80) takes form [15]

$$
V\left(\rho, \rho_{0}\right)=2 \frac{\rho^{8}\left(\rho^{4}-\rho_{0}^{4}\right)-\rho_{0}^{4}\left(4 \rho^{4}-1\right)-3 \rho^{4}}{\rho^{6}\left(\rho^{4}-1\right)}, \quad 1<\rho_{0} \leq \rho<\infty .
$$

The behavior of this potential for different values of $r_{0}$ is shown in figure 25, Unlike the $A d S_{5} \times S^{5}$ case, there exist regions where the potential becomes negative, this is in agreement with the results of section 4.2 where negative eigenmodes where found. The potential starts from a negative value at $\rho_{0}$ given by $V\left(\rho_{0}, \rho_{0}\right)=-8 / \rho_{0}^{2}$. As $\rho_{0}$ increases the negative region gets dimmer and the negative modes cease to exist at the critical value, found numerically in section 4.2, $\rho_{0 c} \simeq 1.177$ which precisely coincides with the critical value of the length function $L\left(\rho_{0}\right)$. We conclude that the classical embeddings satisfying $L^{\prime}\left(\rho_{0}\right)>0$ are unstable under linear perturbations (see also [15] for a perturbative analysis of the eigenvalues). The classical unstable solutions $L^{\prime}\left(\rho_{0}\right)>0$ have regularized energy $E_{q \bar{q}}$ greater than zero (see fig. 6), since the reference configuration satisfies the same boundary conditions, the natural candidate for the decay process is the reference (free quarks) state.

For completeness we quote that since the asymptotics of this background coincides with the previous case, the Schrodinger equation for in-plane fluctuations results defined on a finite interval. The spectrum of stable fluctuations is therefore discrete.

\subsection{Maldacena-Núñez}

The Schrodinger potential for (81) takes the form

$$
V\left(r, r_{0}\right)=\frac{e^{-2 \phi(r)}}{4}\left(\left(e^{2 \phi(r)}-3 e^{2 \phi\left(r_{0}\right)}\right) \phi^{\prime 2}(r)+2\left(e^{2 \phi(r)}+e^{2 \phi\left(r_{0}\right)}\right) \phi^{\prime \prime}(r)\right), \quad 0<r_{0} \leq r<\infty .
$$




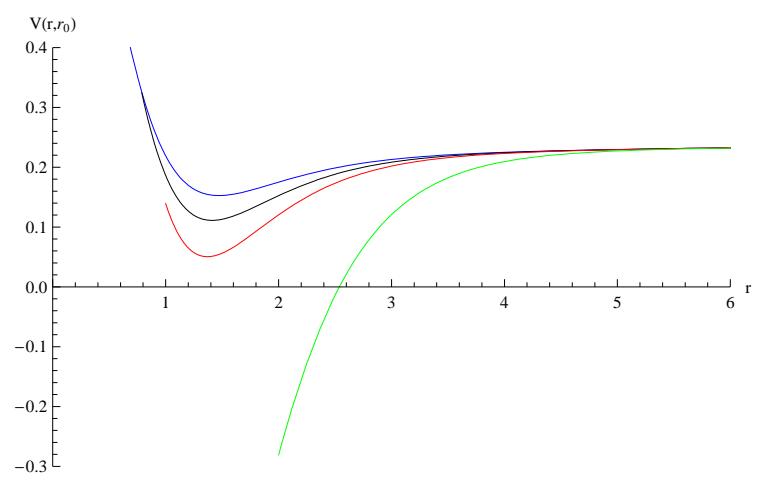

Figure 26: Schrodinger potential (94). The blue, black, red and green lines corresponds to $r_{0}=0.2,0.7,1,2$. The minimum of the potential becomes negative for $r_{0} \geq 1.1605$.

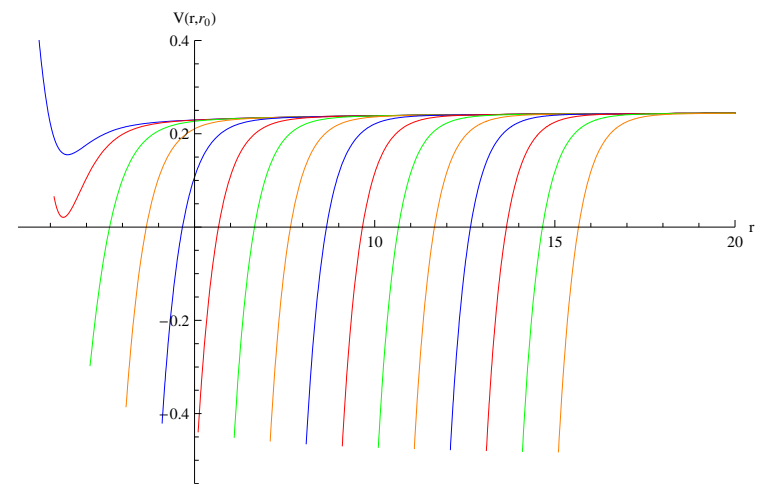

Figure 27: Potential (94) as a function of $r_{0}$. The potential asymptotically tends to $V_{\infty}=\frac{1}{4}$ in agreement with (83). For $r_{0}<1.1605$ is positive negative and for $r_{0} \geq 1.1605$ contains negative regions. Near the tip the potential can be approximated asymptotically (for large $\left.r_{0}\right)$ by $V(r) \simeq-\frac{1}{2}+\frac{3}{2}\left(r-r_{0}\right)$

As before $r$ should be understood as $r=r(y)$ and contrary to the last two cases the change of variables (105) give a Schrodinger problem in $y$ coordinates defined on the half line $y \in[0, \infty)$. Figures [26] and 27] show the Schrodinger potential (94) for different values of $r_{0}$. We should confront these figures with the results in section 4.3. The outcome of that section, for all values of $r_{0}$, was that a continuum spectrum results for $\omega^{2}>0$ and numerically no negative normalizable modes were found. We first address the continuum spectrum for $\omega^{2}>0$. Figure 26] shows that the potential is positive definite for $r_{0} \leq 1.1605$ and asymptotes the value $V_{\infty}=\frac{1}{4}$. One might therefore conclude no solutions for $0<\bar{\omega}^{2}<V_{\min }$, a discrete spectrum for $V_{\text {min }}<\bar{\omega}^{2}<\frac{1}{4}$ (if possible) and a continuum, but not normalizable, spectrum for $\bar{\omega}^{2}>\frac{1}{4}$, all in contradiction with the mentioned results. The agreement is achieved when taking into account the factor $(P Q)^{-\frac{1}{4}}$ that relates the solution of the Schrodinger equation $\Psi$ with the fluctuation $\delta x_{1}$ (see appendix $\mathrm{A}$ eqn. (105)

$$
\delta x_{1}=\frac{e^{\frac{\phi(r)}{2}}}{\left(e^{2 \phi(r)}-e^{2 \phi\left(r_{0}\right)}\right)^{\frac{1}{2}}} \Psi \simeq e^{-\frac{r}{2}} \Psi, \quad r \rightarrow \infty .
$$

The $e^{-\frac{r}{2}}$ factor makes all $\bar{\omega}^{2}>0$ solutions of the Schrodinger problem satisfy the $\left.\delta x_{1}\right|_{r=\infty}=0$ whether or not they normalizable as $\Psi(y)$ solutions (asymptotically one has $y \simeq r$ ). However, for $\bar{\omega}^{2}<0$ solutions the factor is not enough for making the (diverging) solutions satisfy the boundary condition. We conclude that for all $r_{0}$ a continuum spectrum results for $\bar{\omega}^{2}>0$.

The remaining point to be addressed is the possibility of bound states for $-\frac{1}{2}<\bar{\omega}^{2}<0$ in the limit of large $r_{0}$. As seen from figure 27, asymptotically, the potential starts from $V\left(r_{0}\right) \simeq-\frac{1}{2}$ and the linear approximation one obtains is $V(r) \simeq-\frac{1}{2}+\frac{3}{2}\left(r-r_{0}\right)$. The relation between the $r$ and $y$ coordinates (105) in the same limit is $\left(r-r_{0}\right) \simeq y^{2} / 2$. All these leads to a harmonic oscillator in $y$ coordinates with bound state energy above zero. We therefore conclude that no bound states exist. We find a complete agreement between the Schrodinger analysis and the numerical results of section 4.3 .

The results are appealing since if instabilities were found, no obvious candidate for the decay is available (cf. section 5.5). 


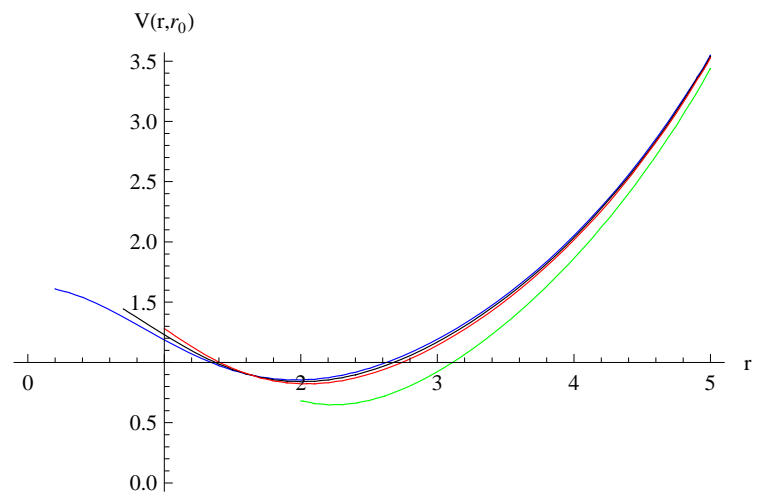

Figure 28: Schrodinger potential (96). The blue, black, red and green lines corresponds to $r_{0}=0.2,0.7,1,2$. The potential is positive definite and therefore no negative (unstable) eigenvalue modes result.

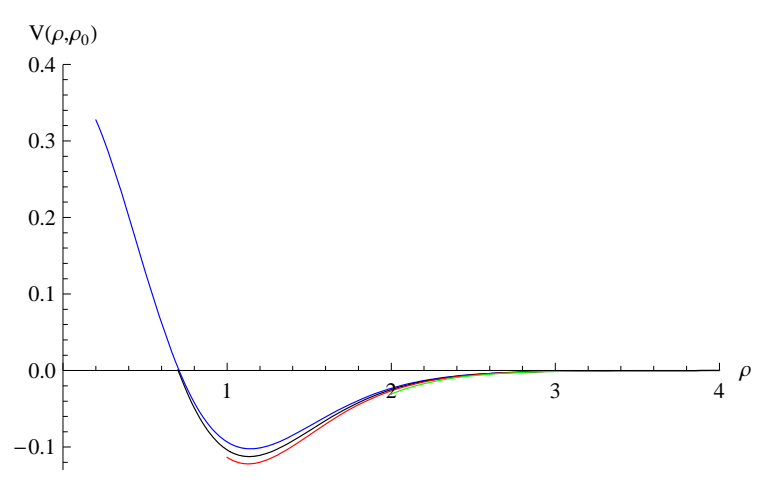

Figure 29: Schrodinger potential (97) for different $\rho_{0}$ values and $\mu=-1$. The blue, black, red and green lines correspond to $\rho_{0}=$ $0.2,0.7,1,2$ respectively.

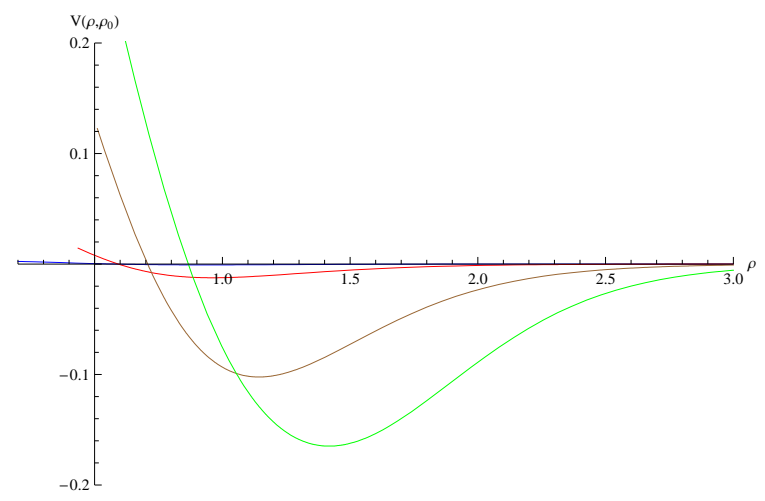

Figure 30: Schrodinger potential for $\rho_{0}=0.2$ and different values of the parameter $\mu$. The blue, red, brown and green lines correspond to $\mu=-1.8,-1.5,-1,-0.8$.

\subsection{Klebanov-Strassler}

The potential for the Klebanov-Strassler in-plane fluctuation (84) reads

$$
\begin{aligned}
V\left(r, r_{0}\right)= & -\frac{3 K(r)}{8 h^{3}(r) h\left(r_{0}\right)}\left[4 h(r)\left(h(r)+h\left(r_{0}\right)\right) h^{\prime}(r) k^{\prime}(r)\right. \\
& \left.\left.-k(r)\left(3 h(r)+7 h\left(r_{0}\right)\right) h^{\prime 2}(r)+4 h(r)\left(h(r)+h\left(r_{0}\right)\right) h^{\prime \prime}(r)\right)\right]
\end{aligned}
$$

The asymptotic behavior of the $P, Q$ functions in this case lead to a Schrodinger problem defined on a finite $y$ interval (see appendix $\mathbf{A}$ ). Figure (28) shows the form of the potential for various $r_{0}$ values. The potential is positive definite and therefore no (unstable) $\omega^{2}<0$ solutions exist. The finite interval on which the Schrodinger problem is defined implies a discrete set of eigenvalues. We find complete agreement with the results of section 4.4 .

\subsection{Generalized Maldacena-Núñez}

The Schrodinger potential for in-plane fluctuations in the generalized MN solutions (88) can be written as

$$
V\left(\rho, \rho_{0}\right)=\frac{2}{e^{8 f(\rho)+2 k(\rho)}}\left(2\left(e^{8 f(\rho)}-e^{8 f\left(\rho_{0}\right)}\right) f^{\prime 2}(\rho)+\left(e^{8 f(\rho)}+e^{8 f\left(\rho_{0}\right)}\right)\left(f^{\prime \prime}(\rho)-k^{\prime}(\rho) f^{\prime}(\rho)\right)\right)
$$


The asymptotic behavior of the $P, Q$ functions (see appendix $A$ and eqn. (89)) leads to a Schrodinger problem formulated on the half line $y \in[0, \infty)$. Figure (29) shows the behavior of the potential for different values of $\rho_{0}$ and a fixed value of $\mu=-1$. The potential becomes negative above some critical value $\rho_{*}$ and asymptotes $V_{\infty}=0$ in concordance with (89) and the existence of negative (unstable) modes found numerically in section 4.5. The $(P Q)^{-\frac{1}{4}}$ factor relating the Schrodinger wave function $\Psi$ to the fluctuation $\delta x_{1}$ approaches a constant at infinity, therefore not changing the asymptotics of the $\Psi$ solutions (cf sect. 5.3). Figure 30 shows the Schrodinger potential, for a fixed value of $\rho_{0}=0.2$, for different values of $\mu$. The minimum of the potential decreases as the $\mu$ approaches $-\frac{2}{3}$. As already mentioned the original MN solution (47) is not continuosly connected with the generalized class of solutions (63). Agreement with the numerical results of section 5.5 is found but it is no clear to us which is the final state of the decay.

\section{6 't Hooft loop}

The electromagnetic dual to Wilson lines in Yang-Mills theories are the 't Hooft lines [35]. In four dimensions, the mechanism for confinement is supposed to be due to magnetic monopole condensation (dual Meissner effect), the analysis in 35] concluded that a screened monopole potential between a $m \bar{m}$ pair should be observed when confinement is due to a dual Meissner effect. A generalization of this idea is dyon confinement and goes under the name of oblique confinement.

The string prescription for computing 't Hooft loops in the MN and KS solutions was proposed in the same papers [25]-[26] (see [36] and also [31] where a technical issue, correcting the proposed 2-cycle in [25], was pointed out) and consists in wrapping a probe $D 3$-brane on the same 2-cycle on which the $D 5$-branes leading to the backreacted geometry were wrapped (see also [9]). The outcome of the construction is an effective D1-brane (string) which is analyzed in complete analogy with the probe fundamental string we have been discussing in previous sections. The important difference with respect to the Wilson loop case is that the 't Hooft loop in generic non S-dual theories is sensible to the internal five dimensional manifold.

\subsection{Maldacena-Núñez}

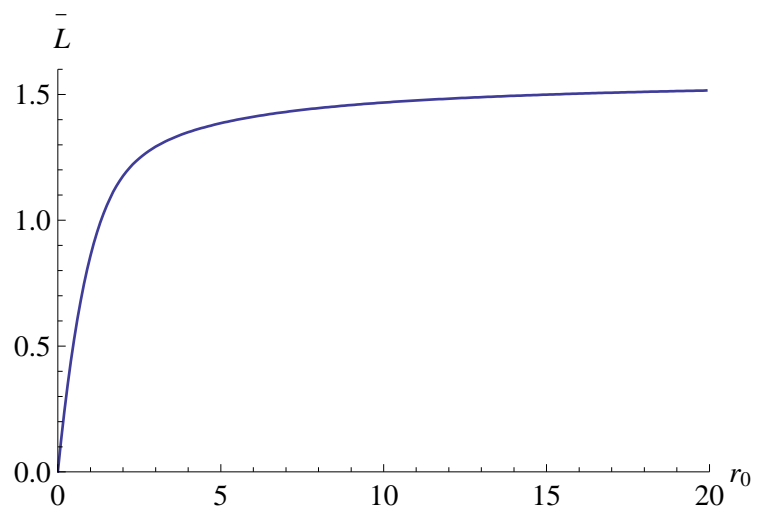

Figure 31: The length function for the effective string as a function of $r_{0}$ in the Maldacena-Núñez t Hooft loop case.

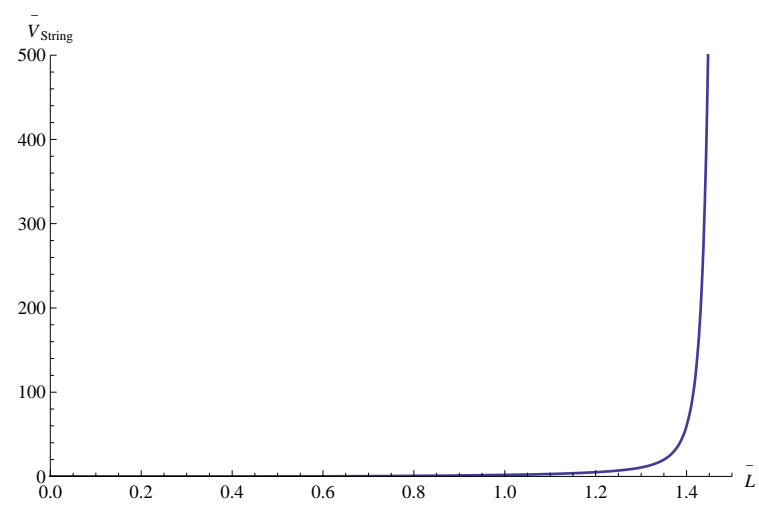

Figure 32: The energy of the monopoleantimonopole pair as a function of the separation length.

The embedding manifold for the $D 3$ in the metric (47) is $[36]$

$$
\mathcal{M}_{4}=[t, x, r(x), \theta=\tilde{\theta}, \varphi=2 \pi-\tilde{\varphi}, \psi=\pi] .
$$

\footnotetext{
${ }^{10}$ The remaining coordinates are set to constants. The value of the $\psi$ coordinate is fixed by demanding the $S^{2}$ to be of minimal volume.
} 
The induced metric on $\mathcal{M}_{4}$ results in

$$
d s_{i n d}^{2}=\alpha^{\prime} N e^{\phi}\left[-d t^{2}+\left(1+\dot{r}^{2}\right) d x^{2}+\left(e^{2 h}+\frac{1}{4}(1-a)^{2}\right)\left(d \theta^{2}+\sin ^{2} \theta d \varphi^{2}\right)\right],
$$

and the expressions (49) for $a, h$ give

$$
V_{S^{2}}(r) \equiv \frac{1}{4}(1-a(r))^{2}+e^{2 h(r)}=r \tanh r .
$$

Note that the $S^{2}$ sphere smoothly collapses at the origin. Integrating the DBI action 11

$$
S_{D B I}=-T_{D 3} \int d^{4} \sigma e^{-\phi} \sqrt{g_{\text {ind }}}
$$

over the internal manifold $\left(S_{\theta \varphi}^{2}\right)$ and the time coordinate one obtains

$$
S_{\text {eff }}=4 \pi T_{D 3} \mathcal{T}\left(\alpha^{\prime} N\right)^{2} \int e^{\phi} r \tanh r \sqrt{1+\dot{r}^{2}} d x .
$$

The important difference wrt the previous Wilson loop calculation in the Maldacena-Núñez background (see sect. (3.3) resides in the $f(r)$ and $g(r)$ functions in (50)-(51) being multiplied by the 2 -sphere volume (100).

In figures 31 we plot the behavior of the length function (9) as a function of $r_{0}$. The length function is an increasing function of $r_{0}$ and from the previous discussions we therefore expect the embedding to be unstable. The instability of the embedding can be easily seen in the Maldacena-Núnez case since a fluctuation along the $x_{1}$-direction depending only on $t, r$ is decoupled from the angular ones (consistent fluctuation). The $\delta x_{1}$ equation of motion results in (22) with $f(r)=g(r)=h(r)=r \tanh r e^{\phi(r)}$. The asymptotics of the fluctuation is the same as in the Wilson loop case, nevertheless the behavior drastically changes near the origin since $f(r)$ goes to zero. As seen in figure 33 negative eigenvalues exist for all $r_{0}$ values. For completeness we plot in figure 34 the Schrodinger potential associated with the in-plane fluctuation equation of motion.

In figure 32 we plot the energy as a function of the endpoints separation length $L$. The energy of the configuration is positive for all $L$, this fact and the instability of the configuration suggests that the stable configuration for given boundary conditions is the one corresponding to two "straight lines". Contrary to the Wilson loop case the "straight lines" (used as reference state for regularizing the energy) can end at the origin since they correspond to wrapped $D 3$ on the topological $S^{2}$ of (47) which smoothly collapses at the origin.

\subsection{Klebanov-Strassler}

This case again involves wrapping a D3 over a topological $S^{2}$ inside (54) (for its parametrization see the appendix A of [37]). There are important differences with respect to the MN case, in the present case the $H_{3}$ supporting the KS geometry contributes to the string action (1), and moreover it leads to the entanglement of the angular and the in-plane fluctuations. The different UV behavior wrt the MN is the reason for the length function $L\left(r_{0}\right)$ having a priori stable regimes (see figure 35). The behavior of $\bar{V}(\bar{L})$ shows the potential is screened for large $L$ and this agrees with the linear confinement potential for the Wilson loop case (see [9] for a related example). The analysis of the present case is analogous to the one in sect. 3.2, summarizing when the configuration energy becomes positive, the two "straight lines" solution becomes favored. As for the MN case this becomes possible without a horizon in this case due to the $D 3$ being wrapped over a smoothly collapsing $S^{2}$ at the origin. We did not attempt the analysis of the coupled fluctuations equations of motions to check for instabilities on the left branch of fig. 35 .

\footnotetext{
(7).

${ }^{11}$ Placing the gauge fixed ansatz (98) into the action (101) give the correct equation of motion for $r(x)$ which coincides with
} 


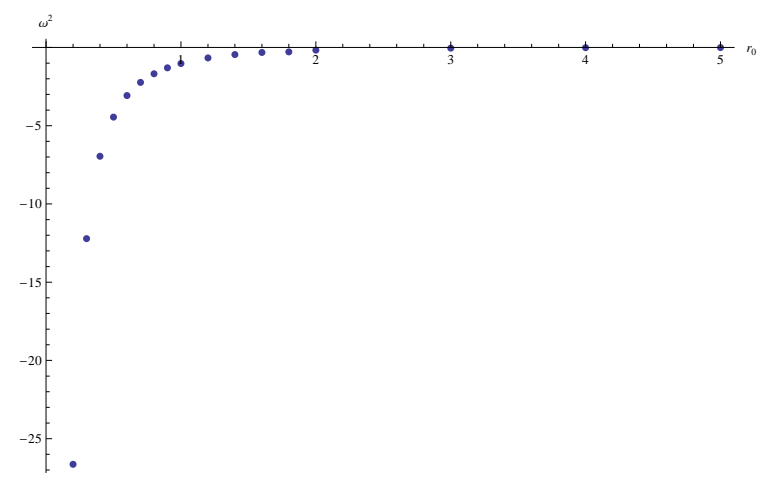

Figure 33: Lowest numerical eigenvalue $\omega^{2}$ for in-plane fluctuations depending only on $t, r$ coordinates as a function of $r_{0}$ in the MaldacenaNúñez 't Hooft loop case.

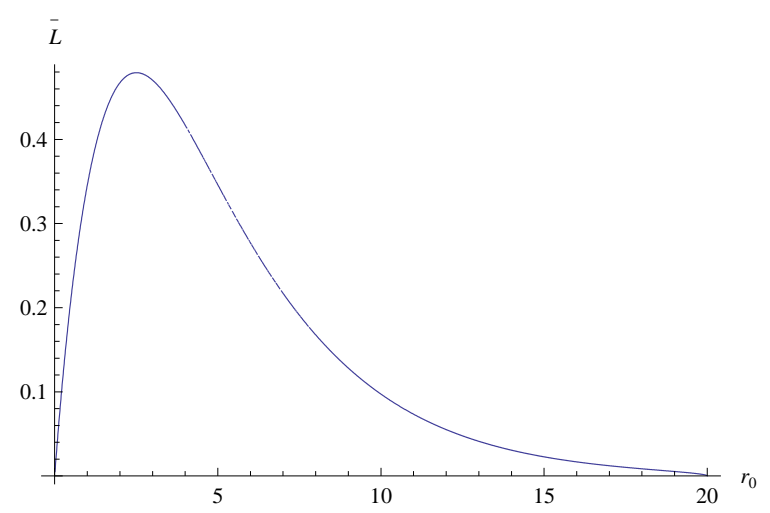

Figure 35: Length function $L\left(r_{0}\right)$ for the effective string as a function of $r_{0}$ in KS background.

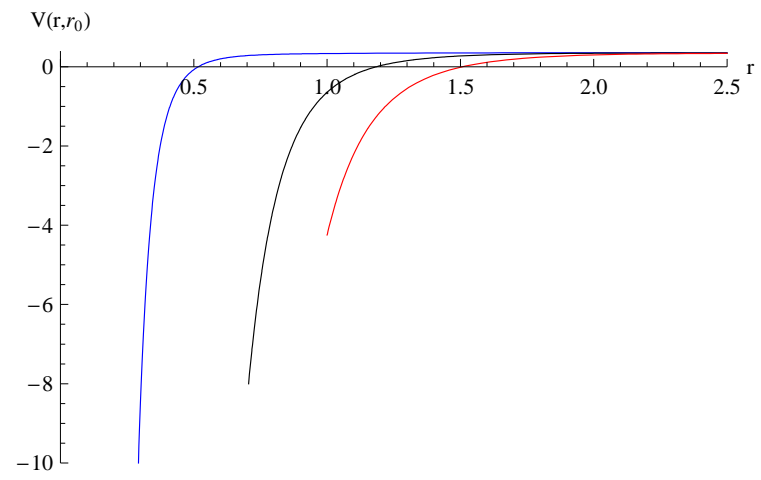

Figure 34: Schrodinger potential for in-plane fluctuations depending on $t, r$-coordinates as a function of $r_{0}$ in the Maldacena-Núnez t Hooft loop case. Blue, black and red lines correspond to $r_{0}=0.2,0.7,1$.

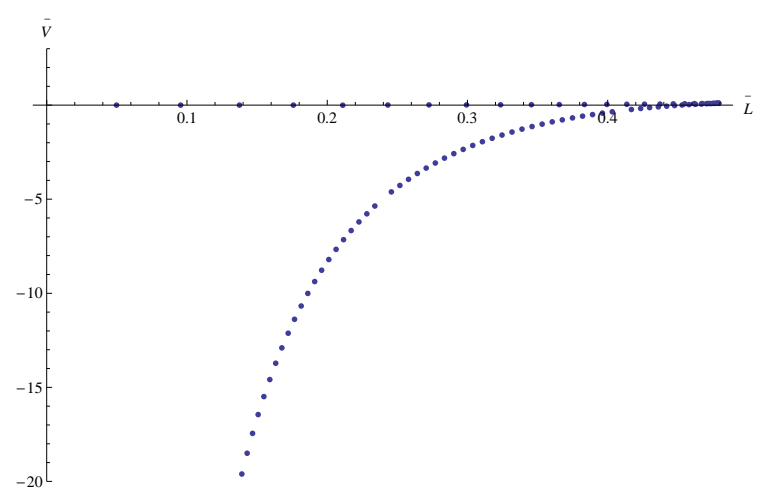

Figure 36: The energy of the monopoleantimonopole pair as a function of the separation length.

\section{Conclusions}

In this work we have analyzed the string proposal for computing rectangular Wilson loops via string embeddings in gravity backgrounds and we have studied their stability under linear perturbations.

The string prescription involves solving for a minimal open string worldsheet whose endpoints lie on the loop to be computed located at a fixed value of the holographic radial direction. When the endpoints are moved to infinity a divergent area results and a regularization is mandatory in order to get a meaningful answer. In section 2 we have reviewed this prescription and showed how a finite value is obtained. We have chosen to regularize the action by the standard procedure originally proposed in [4]. This is interpreted as saying that the Nambu-Goto worldsheet area computation includes the interaction energy plus the self energy (mass) of the external quarks. Within this interpretation we reproduced the well known results for $A d S$ and thermal $A d S$. The regularization was in fact responsable for turning the original positive area into a negative attractive potential energy. When turning to smooth backgrounds ( $A d S$ in global coordinates, MN and KS) a puzzle arises since the straight strings running along the radial direction used in the substraction prescription must end somewhere in the bulk. We concluded that the correct interpretation for the substraction procedure is that we are comparing the string 'Wilson loop' worldsheet with respect to a reference state consisting in a straight string worldsheet whose endpoints lie at antipodes of a compact direction (pictorically represented 
in fig. (10). It then follows that the reference state in general satisfies different boundary conditions than the worldsheet used in computing the expectation value for the rectangular Wilson loop. This last observation is welcomed for the MN and KS cases where the linear confining relation occurs for worlsheets having positive regularized energies (see figs. 9 and 13): if the reference state satisfied the same boundary conditions as the 'Wilson loop' worldsheet, the observed linear behavior should not be considered since the reference state $\left(E_{q \bar{q}}=0\right)$ would have been the lowest energy one (cf. last paragraph of sect. 3.2), but from the previous analysis we see that this is not the case. We would like to recall an observation in [9] stating that the relation between Wilson loops and strings in gravity duals (at the semiclassical level)

$$
\langle W\rangle \simeq e^{-A}
$$

is schematic since the addition of boundary terms to the Nambu-Goto action does not change the minimal area character of the solutions but turns the value of the classical action into something different than the area. In [9] this arbitrariness was used to make a Legendre transform of the Nambu-Goto action showing that the resulting quantity, for the case of loops in $A d S$, is free from the linear divergences arising from the behavior of the worldsheet near the boundary of $A d S$.

We also discussed the concavity conditions (17) that must be satisfied by any potential pretending to describe the interaction between physical quarks. Generic gravity duals have positive and increasing $f(r)$ functions, so the concavity conditions are not satisfied when the length function is an increasing function of the minimal radial position reached by the string $r_{0}$. In section 3 we performed the analysis of the length and potential functions $L\left(r_{0}\right)$ and $V_{\text {string }}(L)$ for different backgrounds and showed that some of them lead to embedding solutions where the concavity condition fails.

Based on previous work [14]-[15] we studied linear fluctuations around the embedding to test the stability of the classical embedding. We concluded that whenever the solution leads to an unphysical potential, not satisfying the conditions (17) there exist unstable modes under linear fluctuations. In the course of the analysis we discussed the different gauge fixings that can be imposed and its relation with the diffeomorphism of the Nambu-Goto action. Three natural gauge fixings where discussed and we chose to work in the $r$-gauge since it lead to simpler closed expressions for the fluctuations equations of motion (see eqn. (21)). The $r$-gauge leads to singular behavior in the fluctuation at the tip of the embedding, but reviewing [15] we showed that nevertheless they are physical once an appropriate gauge transformation is performed.

In section 4 we perform the stability analysis for the solution reviewed in section 3 . We showed by a numerical analysis that the $A d S_{5} \times S^{5}$, Maldacena-Núñez and Klebanov-Strassler are stable. On other hand for thermal $A d S$ and the generalized Maldacena-Núñez backgrounds of sect. 3.5 we found unstable modes in agreement with the behavior of the $L\left(r_{0}\right)$ relation. This last case is rather pathological since the loop cannot be placed at infinity and moreover we found that a minimum separation exists beyond which no smooth solution connecting the string endpoints exists. In section 5 transforming the Sturm-Liouville fluctuation equations of motion into a Schrodinger like equation we reanalyzed the problem, finding complete agreement with the results obtained in section 4 . We conclude that the regions where we find unstable modes coincide with the regions where the concavity condition fails.

In the last section we performed the previous analysis for the case of monopole-antimonopole interaction in the non-conformal gravity duals of Maldacena-Núñez and Klebanov-Strassler. We discussed the 't Hooft loop string prescription given by wrapping a $D 3$ on the topological $S^{2}$ present in the geometries. The MN case was shown to be unstable for all $r_{0}$ values. A fluctuation analysis was feasible since a decoupled equation for the in-plane fluctuation could be found were an unstable mode was shown to exist by a numerical analysis. The KS presented a behavior similar to thermal $A d S$ with presumably stable and unstable regions, but a the fluctuation analysis lead to coupled fluctuations equations of motion which we did not analyze.

We conclude that the analysis of Wilson/'t Hooft loops in given gravity background by looking at the value of the $f^{2}$ at the origin should be supplemented with an analysis of the $L\left(r_{0}\right)$ relation.

\section{Acknowledgments}

We thank D. Arean, N. Grandi, A. Lugo, J. Maldacena, M. Schvellinger, M. Sturla for helpful discussions and correspondence. We are specially grateful with C. Nuñez for a careful reading of the manuscript and sharing 
with us a draft of [33. This work was partially supported by PIP6160-CONICET and by the ANPCyT PICT-2007-00849.

\section{A Sturm-Liouville to Schrödinger}

Equations (22)-(23) are of the Sturm-Liouville type

$$
\left[-\frac{d}{d r}\left(P\left(r, r_{0}\right) \frac{d}{d r}\right)+U\left(r, r_{0}\right)\right] \Phi(r)=\omega^{2} Q\left(r, r_{0}\right) \Phi(r), \quad r_{0} \leq r<\infty
$$

the functions $P\left(r, r_{0}\right)$ and $Q\left(r, r_{0}\right)$ can be read off from (22) $-(\underline{23}), U\left(r, r_{0}\right)=0$ in both cases. The change of variables

$$
y=\int_{r_{0}}^{r} \sqrt{\frac{Q}{P}} d r, \quad \Phi(r)=(P Q)^{-\frac{1}{4}} \Psi(y)
$$

transforms (104) to a Schrödinger like equation

$$
\left[-\frac{d^{2}}{d y^{2}}+V\right] \Psi=\omega^{2} \Psi, \quad 0 \leq y \leq y_{0} .
$$

Here $y_{0}=\int_{r_{0}}^{\infty} d r \sqrt{\frac{Q}{P}}$ which may be finite or infinite depending on the nature of $Q, P$ and one can check that (105) is integrable at the lower limit giving $y \sim \sqrt{r-r_{0}}$. The potential $V$ is given by

$$
\begin{aligned}
V & =\frac{U}{Q}+\left[(P Q)^{-\frac{1}{4}} \frac{d^{2}}{d y^{2}}\right](P Q)^{\frac{1}{4}} \\
& =\frac{U}{Q}+\left[\frac{P^{\frac{1}{4}}}{Q^{\frac{3}{4}}} \frac{d}{d r}\left(\sqrt{\frac{P}{Q}} \frac{d}{d r}\right)\right](P Q)^{\frac{1}{4}}
\end{aligned}
$$

The points $r=r_{0}$ and $r=\infty$ map to $y=0$ and $y=y_{0}$ respectively. The boundary conditions to be imposed on the solutions of (106) are [15]:

- Infinity: string endpoints fixed 12

$$
\left.\delta x\right|_{r=\infty}=\left.0 \Rightarrow \Psi\right|_{y=y_{0}}=0
$$

- Tip $r=r_{0}$ : for both in-plane $\delta x_{1}$ and transverse fluctuations $\delta x_{m}$ one obtains from (24), (31), (32)

$$
\begin{array}{cl}
\text { Even solutions : } & \left.\frac{d \Psi}{d y}\right|_{y=0}=0 \\
\text { Odd solutions : }\left.\quad \Psi\right|_{y=0}=0 .
\end{array}
$$

\section{B Exact spectrum for transverse fluctuations in $A d S_{5} \times S^{5}$}

We review here the solution of [13] for the exact spectrum of the longitudinal fluctuations in the $A d S_{5} \times S^{5}$ background and compare it with our numerical results using the shooting technique described at the end of section 2.2 .

The AdS metric is written in Poincare coordinates

$$
d s^{2}=\frac{R^{2}}{z^{2}}\left(-d t^{2}+d x_{i} d x_{i}+d z^{2}\right)+R^{2} d \Omega_{5}^{2} .
$$

A $x$-gauge fixed ansatz $t=\tau, x=x_{\mathrm{cl}}, z=z_{\mathrm{cl}}(x)$ leads to

$$
\left(\frac{d z_{\mathrm{cl}}}{d x}\right)^{2}=\frac{z_{0}^{4}-\left(z_{\mathrm{cl}}\right)^{4}}{\left(z_{\mathrm{cl}}\right)^{4}} .
$$

\footnotetext{
${ }^{12}$ See however a loophole in the Maldacena-Núñez context (sect. 5.3) when imposing (108).
} 
The solution to (111) with the string endpoints separated by a distance $L$ is (34)-(35)).

$$
x_{\mathrm{cl}}(z)= \pm z_{0}\left[\frac{(2 \pi)^{\frac{3}{2}}}{2 \Gamma\left[\frac{1}{4}\right]^{2}}-\frac{1}{4} \mathrm{~B}\left(\frac{z^{4}}{z_{0}^{4}} ; \frac{3}{4}, \frac{1}{2}\right)\right]
$$

where $z_{0}=z_{\mathrm{cl}}(0)=\left(\Gamma\left[\frac{1}{4}\right]^{2} /(2 \pi)^{\frac{3}{2}}\right) L$ is the maximal radial distance reached by the string (tip of the string).

Fluctuations around the solution (112) in the transverse $x_{m}(m=2,3)$ coordinates decouple, writing $X^{\mu}=\left(t, x_{\mathrm{cl}}(\sigma), \delta x_{m}(t, \sigma), z_{\mathrm{cl}}(\sigma)\right)$ the equations to linear order are [13, [17]

$$
\begin{array}{ll}
x \text {-gauge : } & {\left[\partial_{t}^{2}-\frac{z_{\mathrm{cl}}^{4}(x)}{z_{0}^{4}} \partial_{x}^{2}\right] \delta x_{m}(t, x)=0} \\
r \text {-gauge : } & {\left[\partial_{t}^{2}-\left(1-\frac{z^{4}}{z_{0}^{4}}\right) \partial_{z}^{2}+\frac{2}{z} \partial_{z}\right] \delta x_{m}(t, z)=0 \quad m=2,3 .}
\end{array}
$$

As mentioned in section 2.1, note that the $x$-gauge equation of motion (113) depends explicitly on the classical solution $z_{\mathrm{cl}}(x)$. The equations are related by the change of variables given in (112). Writing $\delta x_{m}=e^{-i w t} f(z)$ in (114) and calling $\tilde{z}=z / z_{0}$ one obtains [17]

$$
\left[\left(1-\tilde{z}^{4}\right) \partial_{\tilde{z}}^{2}-\frac{2}{\tilde{z}}+\xi^{2}\right] f(\tilde{z})=0, \quad 0 \leq \tilde{z} \leq 1,
$$

where $\xi=z_{0} \omega$. The change of variables [38]

$$
\begin{aligned}
f(\tilde{z}) & =\sqrt{1+\xi^{2} \tilde{z}^{2}} F(q) \\
q(\tilde{z}) & = \pm 2 \int_{\tilde{z}}^{1} \frac{t^{2}}{\left(1+(\xi t)^{2}\right) \sqrt{1-t^{4}}} d t
\end{aligned}
$$

transforms equation (115) into a simple harmonic oscillator

$$
\frac{d^{2} F}{d q}+\frac{1}{4} \xi^{2}\left(\xi^{4}-1\right) F=0, \quad q \in\left[-q_{*}, q_{*}\right]
$$

where $q_{*}=q(0)$. The boundary conditions at infinity $\delta x_{m}(t, 0)=0$ have been mapped to $F\left(q_{*}\right)=0$, and quantize the frequencies in (117) leading to

$$
\omega_{n} z_{0} \sqrt{\omega_{n}^{4} z_{0}^{4}-1} \int_{0}^{1} \frac{t^{2} d t}{\left(1+w_{n}^{2} z_{0}^{2}\right) \sqrt{1-t^{4}}}=\frac{n \pi}{2}, \quad n=1,2, \ldots
$$

The following table shows the comparison between the exact eigenvalues (118) and our numerical calculation of eigenvalues of (115) with $z_{0}=1$.

\begin{tabular}{|l||c|c|}
\hline & Exact & Numeric \\
\hline$\omega_{1}$ & 2.203 & 2.226 \\
$\omega_{2}$ & 3.467 & 3.492 \\
$\omega_{3}$ & 4.697 & 4.735 \\
$\omega_{4}$ & 5.914 & 5.959 \\
$\omega_{5}$ & 7.125 & 7.181 \\
$\omega_{6}$ & 8.332 & 8.396 \\
$\omega_{7}$ & 9.537 & 9.612 \\
$\omega_{8}$ & 10.741 & 10.823 \\
\hline
\end{tabular}

The odd (even) eigenvalues where obtained solving (115) with the even (odd) boundary conditions discussed after (24). 


\section{References}

[1] K. G. Wilson, Phys. Rev. D 10 (1974) 2445.

[2] J. M. Maldacena, Adv. Theor. Math. Phys. 2 (1998) 231 [Int. J. Theor. Phys. 38 (1999) 1113] arXiv:hep-th/9711200. S. S. Gubser, I. R. Klebanov and A. M. Polyakov, Phys. Lett. B 428 (1998) 105 [arXiv:hep-th/9802109|. E. Witten, Adv. Theor. Math. Phys. 2 (1998) 253 [arXiv:hep-th/9802150].

[3] A. M. Polyakov, Nucl. Phys. Proc. Suppl. 68 (1998) 1 arXiv:hep-th/9711002]. A. M. Polyakov, Int. J. Mod. Phys. A 14 (1999) 645 arXiv:hep-th/9809057.

[4] S. J. Rey and J. T. Yee, Eur. Phys. J. C 22 (2001) 379 [arXiv:hep-th/9803001]. J. M. Maldacena, Phys. Rev. Lett. 80, 4859 (1998) arXiv:hep-th/9803002.

[5] E. Witten, Adv. Theor. Math. Phys. 2 (1998) 505 arXiv:hep-th/9803131.

[6] G. 't Hooft, Nucl. Phys. B 72, 461 (1974).

[7] J. Sonnenschein, arXiv:hep-th/0003032.

[8] Y. Kinar, E. Schreiber and J. Sonnenschein, Nucl. Phys. B 566 (2000) 103 arXiv:hep-th/9811192.

[9] D. J. Gross and H. Ooguri, Phys. Rev. D 58 (1998) 106002 [arXiv:hep-th/9805129].

[10] C. P. Herzog and I. R. Klebanov, Phys. Lett. B 526, 388 (2002) arXiv:hep-th/0111078.

[11] J. Gomis and F. Passerini, JHEP 0608, 074 (2006) arXiv:hep-th/0604007); JHEP 0701, 097 (2007) arXiv:hep-th/0612022.

[12] S. S. Gubser, Phys. Rev. D 74 (2006) 126005 arXiv:hep-th/0605182.

[13] I. R. Klebanov, J. M. Maldacena and C. B. Thorn, JHEP 0604 (2006) 024 arXiv:hep-th/0602255].

[14] J. J. Friess, S. S. Gubser, G. Michalogiorgakis and S. S. Pufu, JHEP 0704 (2007) 079 arXiv:hep-th/0609137.

[15] S. D. Avramis, K. Sfetsos and K. Siampos, Nucl. Phys. B 769, 44 (2007) arXiv:hep-th/0612139. S. D. Avramis, K. Sfetsos and K. Siampos, Nucl. Phys. B 793 (2008) 1 [arXiv:0706.2655 [hep-th]]. K. Sfetsos and K. Siampos, JHEP 0808 (2008) 071 [arXiv:0807.0236 [hep-th]].

[16] S. J. Rey, S. Theisen and J. T. Yee, Nucl. Phys. B 527 (1998) 171 arXiv:hep-th/9803135. A. Brandhuber, N. Itzhaki, J. Sonnenschein and S. Yankielowicz, Phys. Lett. B 434 (1998) 36 arXiv:hep-th/9803137.

[17] C. G. Callan and A. Guijosa, Nucl. Phys. B 565 (2000) 157 arXiv:hep-th/9906153.

[18] Y. Kinar, E. Schreiber, J. Sonnenschein and N. Weiss, Nucl. Phys. B 583 (2000) 76 arXiv:hep-th/9911123.

[19] S. Forste, D. Ghoshal and S. Theisen, JHEP 9908 (1999) 013 arXiv:hep-th/9903042.

[20] N. Drukker, D. J. Gross and A. A. Tseytlin, JHEP 0004 (2000) 021 arXiv:hep-th/0001204.

[21] P. C. Argyres, M. Edalati and J. F. Vazquez-Poritz, JHEP 0701 (2007) 105 arXiv:hep-th/0608118.

[22] A. Brandhuber and K. Sfetsos, Adv. Theor. Math. Phys. 3 (1999) 851 arXiv:hep-th/9906201.

[23] F. Bigazzi, A. L. Cotrone, C. Nunez and A. Paredes, Phys. Rev. D 78 (2008) 114012 [arXiv:0806.1741 [hep-th]]. F. Bigazzi, A. L. Cotrone, A. Paredes and A. V. Ramallo, JHEP 0903 (2009) 153 arXiv:0812.3399 [hep-th]].

[24] C. Bachas, Phys. Rev. D 33 (1986) 2723. 
[25] J. M. Maldacena and C. Nunez, Phys. Rev. Lett. 86 (2001) 588 arXiv:hep-th/0008001.

[26] I. R. Klebanov and M. J. Strassler, JHEP 0008, 052 (2000) arXiv:hep-th/0007191.

[27] A. Loewy and J. Sonnenschein, JHEP 0108 (2001) 007 arXiv:hep-th/0103163.

[28] R. Casero, C. Nunez and A. Paredes, Phys. Rev. D 73 (2006) 086005 [arXiv:hep-th/0602027]. R. Casero, C. Nunez and A. Paredes, Phys. Rev. D 77 (2008) 046003 [arXiv:0709.3421 [hep-th]].

[29] C. Hoyos-Badajoz, C. Nunez and I. Papadimitriou, Phys. Rev. D 78 (2008) 086005 arXiv:0807.3039 [hep-th]].

[30] C. Vafa, J. Math. Phys. 42 (2001) 2798 [arXiv:hep-th/0008142].

[31] M. Bertolini, Int. J. Mod. Phys. A 18, 5647 (2003) arXiv:hep-th/0303160 ; E. Imeroni, arXiv:hep-th/0312070; A. Paredes, arXiv:hep-th/0407013.

[32] A. H. Chamseddine and M. S. Volkov, Phys. Rev. Lett. 79 (1997) 3343 arXiv:hep-th/9707176;

A. H. Chamseddine and M. S. Volkov, Phys. Rev. D 57 (1998) 6242 arXiv:hep-th/9711181.

[33] C. Nunez, M. Piai and A. Rago, arXiv:0909.0748 [hep-th].

[34] M. J. Strassler, arXiv:hep-th/0505153

[35] G. 't Hooft, Nucl. Phys. B 138 (1978) 1.

[36] M. Bertolini and P. Merlatti, Phys. Lett. B 556 (2003) 80 [arXiv:hep-th/0211142].

[37] C. P. Herzog, I. R. Klebanov and P. Ouyang, arXiv:hep-th/0108101.

[38] R. C. Brower, C. I. Tan and C. B. Thorn, Phys. Rev. D 73 (2006) 124037 arXiv:hep-th/0603256. 